\title{
Holliday Junction Resolvases
}

\author{
Haley D.M. Wyatt and Stephen C. West \\ London Research Institute, Cancer Research UK, Clare Hall Laboratories, South Mimms, \\ Herts EN6 3LD, United Kingdom \\ Correspondence: stephen.west@cancer.org.uk
}

Four-way DNA intermediates, called Holliday junctions (HJs), can form during meiotic and mitotic recombination, and their removal is crucial for chromosome segregation. A group of ubiquitous and highly specialized structure-selective endonucleases catalyze the cleavage of HJs into two disconnected DNA duplexes in a reaction called $\mathrm{HJ}$ resolution. These enzymes, called HJ resolvases, have been identified in bacteria and their bacteriophages, archaea, and eukaryotes. In this review, we discuss fundamental aspects of the HJ structure and their interaction with junction-resolving enzymes. This is followed by a brief discussion of the eubacterial RuvABC enzymes, which provide the paradigm for $\mathrm{HJ}$ resolvases in other organisms. Finally, we review the biochemical and structural properties of some well-characterized resolvases from archaea, bacteriophage, and eukaryotes.

$\mathrm{H}^{2}$ omologous recombination (HR) is an essential process that promotes genetic diversity during meiosis (see Lam and Keeney 2014; Zickler and Kleckner 2014). However, in somatic cells, HR plays a key role in conserving genetic information by facilitating DNA repair, thereby ensuring faithful genome duplication and limiting the divergence of repetitive DNA sequences (see Mehta and Haber 2014). As shown in Figure 1, HR is initiated by a DNA double-strand break, the ends of which are resected to produce single-stranded (ss) $3^{\prime}$ overhangs (see Symington 2014). Homologous strand invasion by one of the $3^{\prime}$ overhangs (e.g., one catalyzed by Escherichia coli RecA or human RAD51) leads to the formation of a displacement loop (D-loop) (see Morrical 2014). The invading $3^{\prime}$ end of the D-loop can then be extended by a DNA polymerase, which uses the homologous strand as a template for DNA synthesis. Recombination then proceeds in one of several different ways, some of which involve second-end capture, such that the other resected $3^{\prime}$ end anneals to the displaced strand of the D-loop (Szostak et al. 1983). In the resulting recombination intermediate, the two interacting DNAs are linked by nicked Holliday junctions (HJs). Additional DNA synthesis and nick ligation lead to the formation of a double Holliday junction $(\mathrm{dHJ})$ intermediate. In eukaryotes, dHJs are removed primarily by "dissolution" (Fig. 1, bottom left) (see Bizard and Hickson 2014). This pathway involves the combined activities of a DNA helicase and a type IA topoisomerase, which catalyze branch migration and decatenation of the $\mathrm{dHJ}$ into noncrossover products (Manthei and Keck 2014). In somatic cells, this is essential for the avoidance of sister-

Editors: Stephen Kowalczykowski, Neil Hunter, and Wolf-Dietrich Heyer

Additional Perspectives on DNA Recombination available at www.cshperspectives.org

Copyright (C) 2014 Cold Spring Harbor Laboratory Press; all rights reserved; doi: 10.1101/cshperspect.a023192

Cite this article as Cold Spring Harb Perspect Biol 2014;6:a023192 
H.D.M. Wyatt and S.C. West
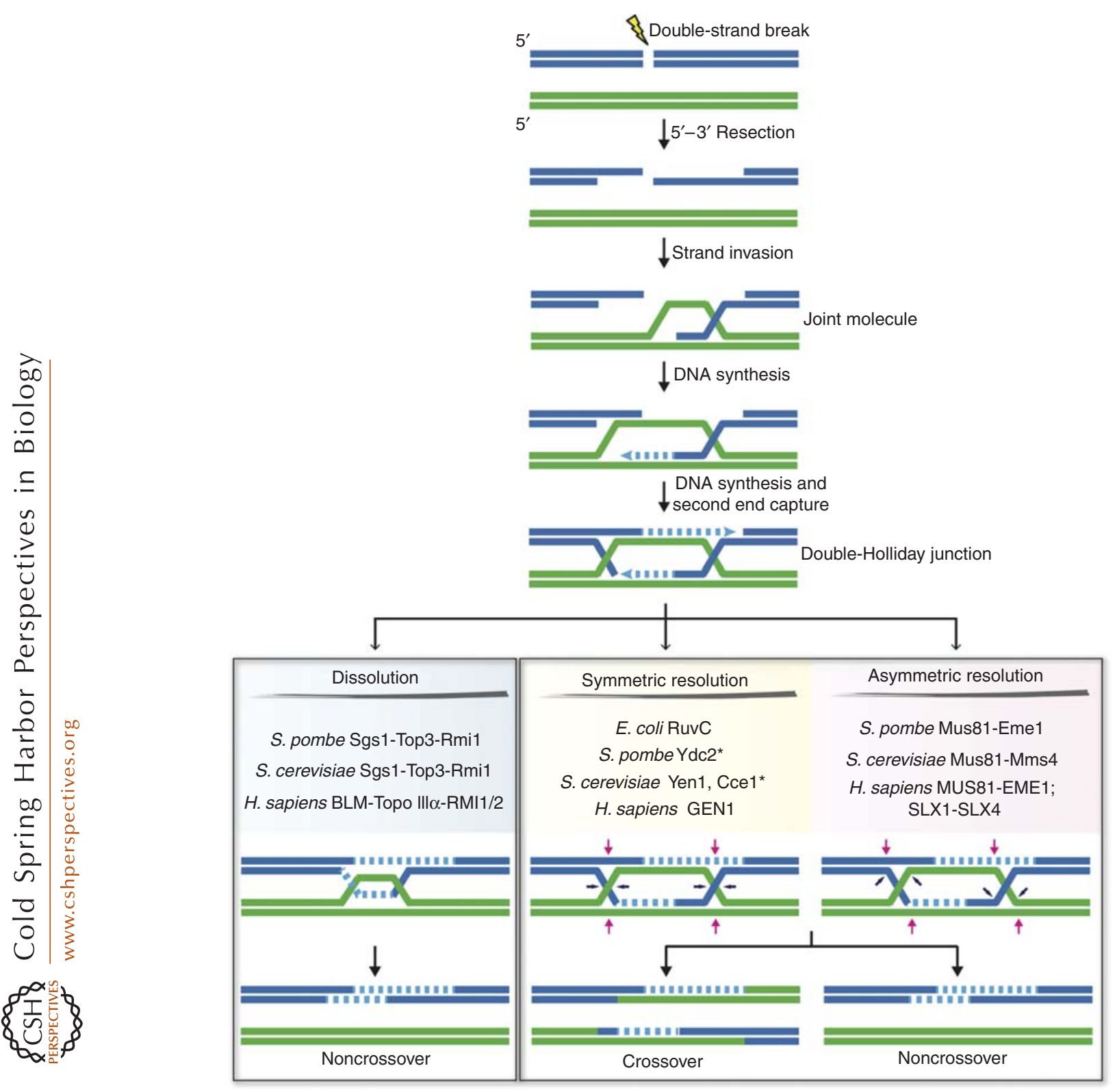

Figure 1. Pathways for the formation and processing of Holliday junctions. Resected DNA double-strand breaks invade homologous duplex DNA to create a joint molecule, or displacement-loop structure. The invading $3^{\prime}$ end then serves as a primer for DNA synthesis, leading to second end capture and the formation of a double Holliday junction. In eukaryotes, these structures are removed by "dissolution" (bottom left panel) or "resolution" (bottom middle and right panels). Canonical Holliday junction resolvases introduce a pair of symmetrical and coordinated nicks across one of the helical axes (bottom middle panel) to generate nicked DNA duplexes that can be directly ligated. Alternatively, noncanonical resolvases cleave Holliday junctions with asymmetric nicks to produce gapped and flapped DNA duplexes that require further processing prior to ligation (bottom right panel). *Mitochondrial Holliday junction resolvase. 
chromatid exchanges (SCEs) and loss of heterozygosity. Alternatively, dHJs can be processed by "resolution" in reactions mediated by canonical or noncanonical mechanisms of endonucleasemediated cleavage into either crossover or noncrossover products (Fig. 1, bottom middle and right).

\section{THE STRUCTURE OF THE HOLLIDAY JUNCTION}

In 1964, Robin Holliday proposed a model to explain three important events that occur during meiosis in fungi: crossing-over, gene conversion, and postmeiotic segregation (Holliday 1964). A central tenet of this model is that after DNA replication, DNA breaks allow pairing between complementary sequences in the two homologous chromosomes, leading to the formation of a cross-stranded structure that physically links the two component helices. The fourway structure at the point of strand exchange later became known as the "Holliday junction" and is now widely accepted to represent a central intermediate in recombination. Our current knowledge regarding the structure of HJs has been obtained using multiple complementary techniques including gel electrophoresis, chemical and enzymatic probing, fluorescence energy transfer, nuclear magnetic resonance, and single-crystal X-ray diffraction studies. These approaches have led to the definition of three general features that are applicable to four-way DNA junctions, each of which are briefly discussed below.

\section{HJs Exist in Open and Stacked} Conformations

Synthetic HJs, made by annealing four complementary oligonucleotides, adopt two global conformations, open and stacked (or unfolded and folded), dependent on the availability and type of divalent metal ions. An excellent indepth review of $\mathrm{HJ}$ structure can be found elsewhere (Lilley 2000). Briefly, in the absence of cations, the negatively charged phosphates are unshielded; this electrostatic repulsion directs the four arms of the junction toward the cor- ners of a square and the junction adopts an unstacked, fully extended conformation with fourfold symmetry (Fig. 2A). The presence of divalent metal ions, however, minimizes electrostatic repulsion, allowing the arms to undergo pairwise coaxial stacking to form a compact $\mathrm{X}$-shaped structure with a twofold symmetry (Fig. 2B-D).

\section{Stacked HJs Have Two Crossover Isomers}

In the presence of divalent metal ions, stacked HJs can exist in two alternative conformations (termed crossover isomers), distinguished by which duplex arms stack on each other (Fig. 2B,C) (Chen et al. 1988, 1993; Duckett et al. 1988). Each isomer comprises two continuous strands on the outer edge of the junction and two exchanging strands on the inner face. This generates two sides with different character; one face has minor groove features and the other has major groove characteristics. The relative stability of the two isomers is generally dependent on the local DNA sequence at the junction (Carlstrom and Chazin 1996; Miick et al. 1997; Grainger et al. 1998).

\section{HJs Exhibit an Antiparallel Geometry}

It was initially believed that HJs would exist in the parallel configuration (Fig. 2B) (Sigal and Alberts 1972). However, biochemical and structural studies revealed that they actually adopt a right-handed antiparallel configuration (Murchie et al. 1989; Clegg et al. 1992; Nowakowski et al. 1999; Ortiz-Lombardia et al. 1999; Eichman et al. 2000). In this orientation, two kinds of strands can be distinguished: (1) two nonexchanging strands of opposite polarity that define the outer face of the HJ, and (2) two exchanging strands that run in opposite directions and pass from one helical axis to the other without physically crossing each other (Fig. 2C,D). The component helices closely resemble Bform DNA, with the exception of a local structural distortion at the point of strand exchange. Base pairing is maintained up to, and including, the point of strand exchange (Wemmer et al. 1985; Chen et al. 1993; Pikkemaat et al. 1994; 
H.D.M. Wyatt and S.C. West

A

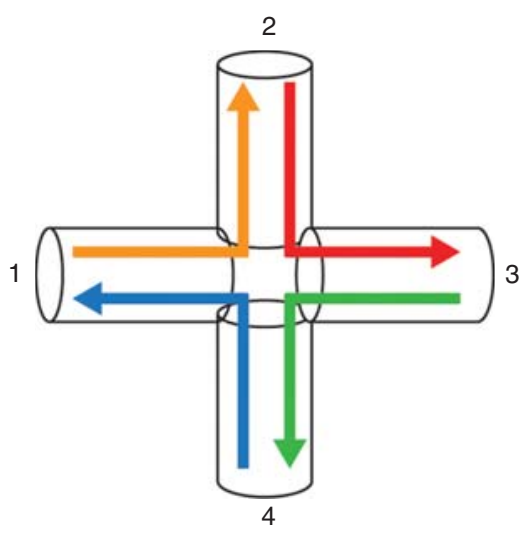

C

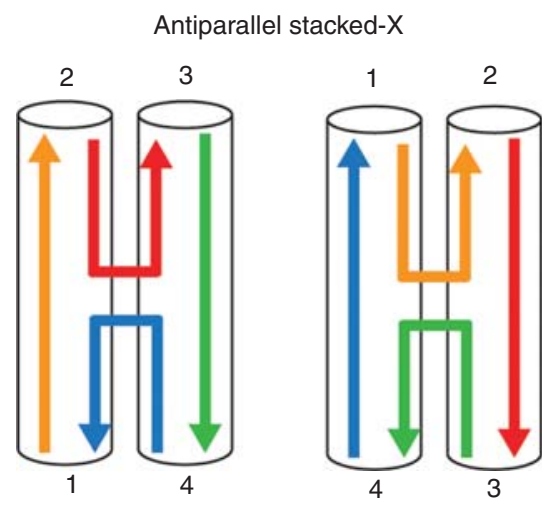

B
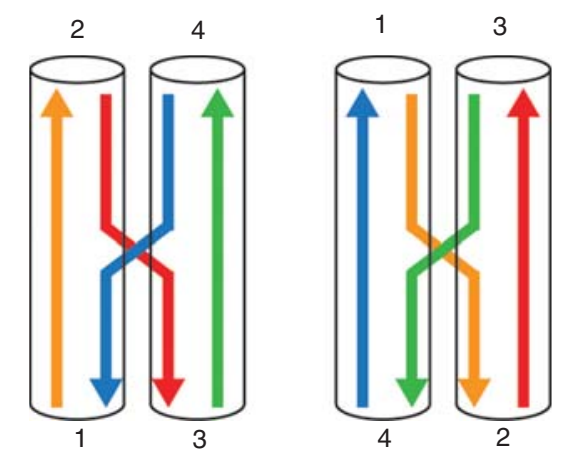

D

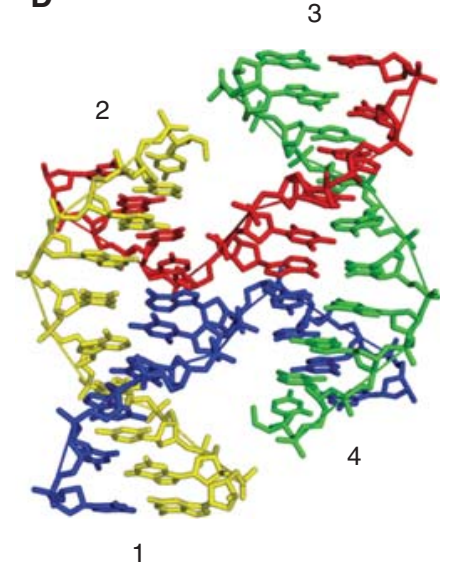

Figure 2. Global conformations of DNA Holliday junctions. The component helices are drawn in different colors and labeled 1 to 4 in a clockwise manner. ( $A$ ) In the absence of divalent cations, four-way junctions adopt a fully extended conformation with no coaxial stacking between the component helices (planar open-X form). Electrostatic repulsion between phosphates keeps the junction in an unstacked conformation with the four arms directed toward the corners of a square. $(B, C)$ In the presence of divalent cations, the helices undergo coaxial stacking such that the symmetry is lowered from fourfold in the fully extended form to twofold in the stacked forms. Pairwise coaxial stacking can occur in two different ways, characterized by the stacking of helices 2 on 1 and 3 on 4 (left) or 1 on 4 and 3 on 2 (right). The stacked X junction can adopt either parallel (B), or antiparallel $(C)$ conformations. $(D)$ Crystal structure of a Holliday junction (Eichman et al. 2000) in the right-handed antiparallel stacked-X conformation (Protein Data Bank, PDB, ID 1dcw), showing the continuous strands in yellow and green and exchanging strands in red and blue.

Carlstrom and Chazin 1996; Overmars and Altona 1997; Overmars et al. 1997). In summary, the $\mathrm{HJ}$ is a dynamic structure that can adopt various conformations. As discussed below, enzymes that promote $\mathrm{HJ}$ resolution exploit this malleability to process the structure into two disconnected DNA duplexes.

\section{JUNCTION RESOLVING ENZYMES}

\section{Hallmark Characteristics}

Holliday junction cleavage, which generates two unconnected DNA duplexes, is catalyzed by a group of structure-selective endonucleases called $\mathrm{HJ}$ resolvases. These nucleases have been 
isolated from bacteria, bacteriophages, archaea, viruses, yeasts, plants, and mammals (Table 1) and share a number of common properties. First, they contain a high proportion of positively charged amino acids, consistent with their ability to bind DNA with high affinity. Second, their active sites generally contain three or four acidic residues required for metal binding and catalysis. Third, a divalent metal ion, usually magnesium $\left(\mathrm{Mg}^{2+}\right)$ or manganese $\left(\mathrm{Mn}^{2+}\right)$, is essential for DNA cleavage, but not for DNA binding. Fourth, HJ resolvases are dimeric enzymes that use twin active sites to catalyze two coordinated incisions within the lifetime of a single protein-HJ complex (Fig. 3). Finally, these enzymes exhibit a preference for binding and cleaving four-way DNA junctions. All recognize the structural distortion that occurs near the helical junction and, on substrate binding, further manipulate the $\mathrm{HJ}$ and open the core to different extents (Fig. 4). This ultimately ensures that the $\mathrm{HJ}$ is resolved into two disconnected DNA duplexes.

\section{General Mechanisms of HJ Resolution}

Recognition of the structural distortion at the helical junction and subsequent opening of the $\mathrm{HJ}$ core is thought to facilitate the placement of DNA in the active sites for subsequent hydrolysis of the phosphodiester backbone (Fig. 3). Canonical $\mathrm{HJ}$ resolvases, such as E. coli RuvC, introduce a pair of coordinated and symmetrical nicks across the helical branch point (Figs. 1 and 3 ). The two nicks occur within the lifetime of a single protein-DNA complex and involve either two simultaneous cleavages or two sequential nicks that occur in rapid succession. Kinetic analyses indicate that first-strand cleavage is rate limiting, whereas second-strand cleavage is accelerated severalfold. It is thought that the first nick relaxes the structure of the $\mathrm{HJ}$ and facilitates placement in the second active site, thus accelerating the second cleavage event. The result of symmetrical resolution is a pair of nicked DNA duplexes, each of which can be directly repaired by the action of a DNA ligase.

In addition to canonical $\mathrm{HJ}$ resolvases, eukaryotes also contain enzymes that catalyze junction resolution by alternative or noncanonical mechanisms. Similar to canonical resolvases, the noncanonical enzymes cleave HJs into duplex DNA via the introduction of two single-stranded incisions. In this case, however, the two nicks are asymmetric and can be located several nucleotides from the branchpoint. In most cases, it is not clear whether these reactions occur within the lifetime of a single protein-DNA complex. The end products are one gapped and one flapped DNA duplex, which require subsequent processing prior to ligation.

\section{HJ Resolvases in Eubacteria}

\section{The RuvABC Proteins}

In E. coli, the ruv locus contains three ruv genes, designated $\operatorname{ruv} A$, ruvB, and $\operatorname{ruv} C$ (Sharples et al. 1990; Takahagi et al. 1991). Mutations in any one of these genes elicit the ruv phenotype: (1) increased sensitivity to ultraviolet (UV) light and mitomycin C (MMC), (2) formation of long, multinucleated filaments after treatment with UV, and (3) moderate defects in HR. This phenotype indicates that the RuvA, RuvB, and RuvC proteins are important for $\mathrm{HR}$ and DNA repair. Indeed, these three proteins form a sophisticated molecular machine, termed the RuvABC resolvasome, which is required for $\mathrm{HJ}$ processing. This complex orchestrates two major events of late-stage recombination: $\mathrm{HJ}$ branch migration (catalyzed by RuvAB) and resolution (catalyzed by RuvC). RuvC is currently the best-characterized cellular resolvase and continues to serve as the paradigm for canonical $\mathrm{HJ}$ resolvases in other organisms.

\section{RuvAB}

RuvA and RuvB were identified and purified in the early 1990s (Iwasaki et al. 1989; Tsaneva et al. 1992a; Shiba et al. 1993) and shown to promote branch migration (Iwasaki et al. 1992; Parsons et al. 1992; Tsaneva et al. 1992b; Parsons and West 1993). RuvA is a tetrameric DNA-binding protein that has a high affinity for HJs. Binding is structure-selective and occurs independently of DNA sequence. Structural studies show that 
H.D.M. Wyatt and S.C. West
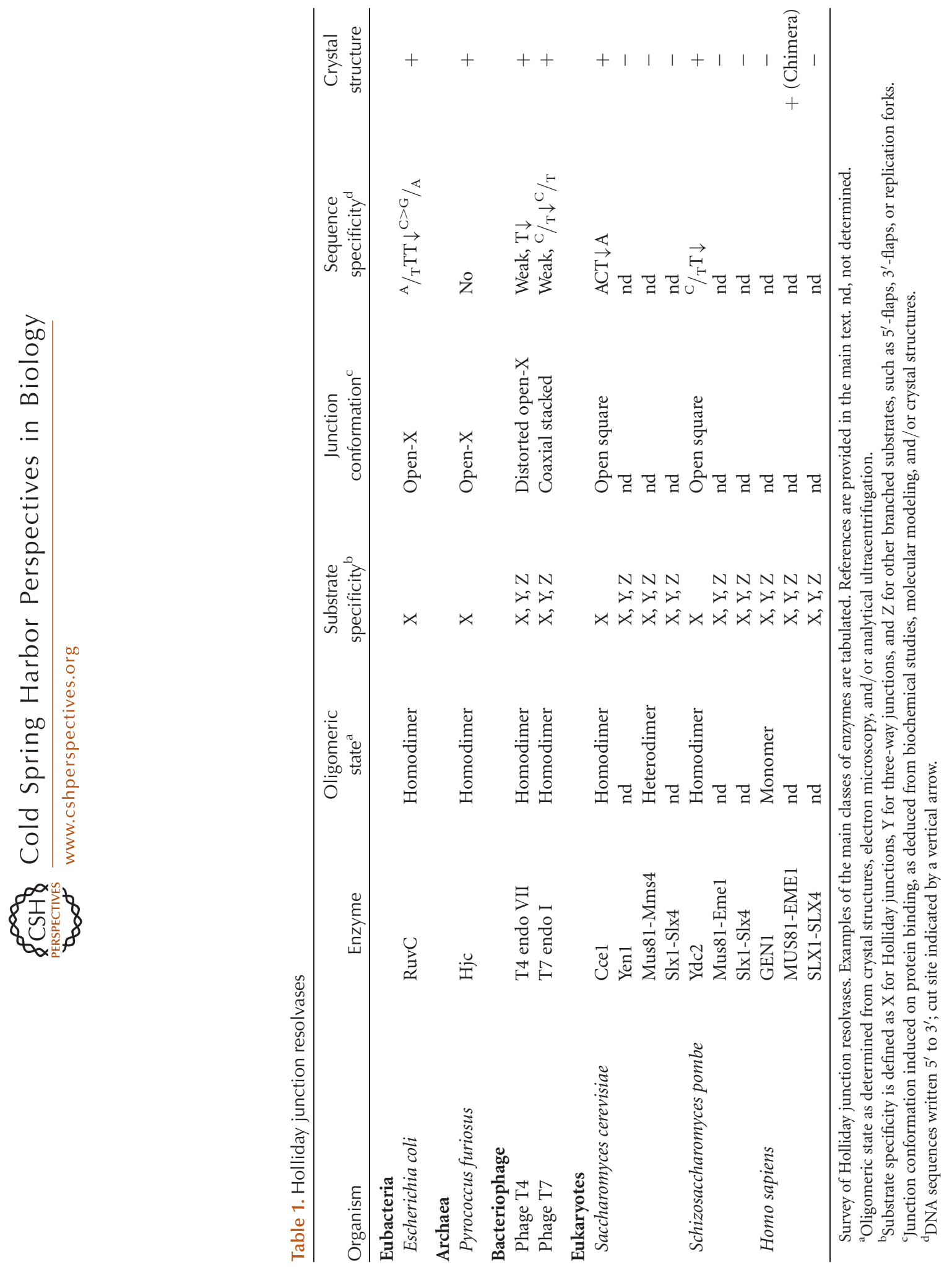
A

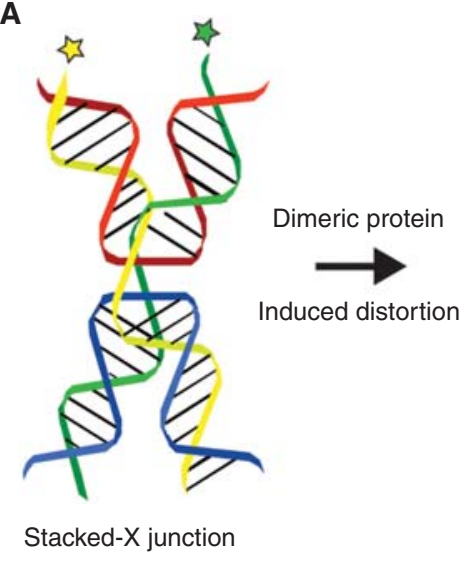

B

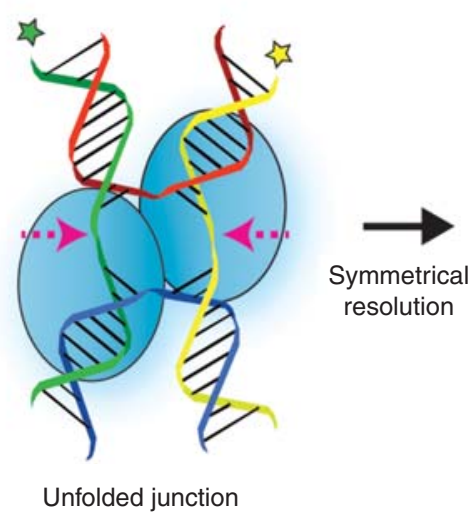

C
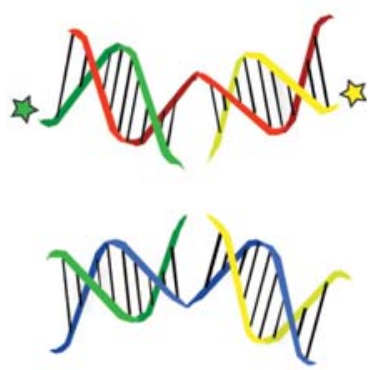

Ligatable nicked DNA duplex products

Figure 3. Canonical mechanism of Holliday junction resolution. (A) Antiparallel stacked-X Holliday junction with twofold symmetry. $(B)$ Canonical Holliday junction resolvases are dimeric enzymes that induce structural changes to the junction on binding, causing the junction to unfold. Resolution occurs by the introduction of two coordinated and symmetrically related nicks in strands of like polarity at, or very near, the branchpoint. $(C)$ Symmetrical resolution gives a pair of nicked DNA duplexes, each of which can be directly repaired by nick ligation. Asterisks signify a given strand of DNA. (From West 2009; adapted, with permission, from the author.)

RuvA exhibits fourfold symmetry and resembles a four-petaled flower (Rafferty et al. 1996; Nishino et al. 1998). Each monomer is composed of three distinct domains. Domains I and II mediate DNA binding, whereas domain III makes direct contacts with RuvB and regulates branch migration (Hargreaves et al. 1998; Nishino et al. 1998; Ariyoshi et al. 2000; Ingleston et al. 2000; Nishino et al. 2000). The tetramer presents two distinct surfaces; one face is convex and has a predominately electronegative charge, whereas the other side is concave and mainly composed of electropositive residues that mediate protein-DNA interactions (Rafferty et al. 1996). Importantly, the concave face contains a central acidic "pin" that is essential for the specific binding to HJ DNA (Ingleston et al. 2000). Moreover, the negative residues repel the phosphate backbones of the four DNA strands at the point of strand exchange and contribute to the disruption of base pairs at the helical junction (Rafferty et al. 1996; Ariyoshi et al. 2000). These interactions are thought to be critical for strand separation during branch migration.

RuvB is a hexameric ring ATPase that exhibits DNA-binding activity (Iwasaki et al. 1992; Tsaneva et al. 1992a; Müller et al. 1993;
Parsons and West 1993; Shiba et al. 1993; Mitchell and West 1994; Marrione and Cox 1995). RuvA facilitates the interaction of RuvB with DNA and stimulates its ATPase activity. The RuvAB-HJ complex is a dumbbell-shaped tripartite structure, in which RuvA is flanked by two oppositely oriented hexameric rings of RuvB (Parsons et al. 1995). The two RuvB motors rotate opposing arms of the open $\mathrm{HJ}$ structure, effectively pumping DNA into and through the RuvAB complex, and, in doing so, convert homoduplex DNA into heteroduplex. The four acidic pins on RuvA act as guides that facilitate unwinding and strand exchange.

\section{RuvC}

The first evidence for a cellular HJ resolvase came from studies demonstrating that fractionated $E$. coli extracts contained an endonuclease activity that (1) resolved recombination intermediates made by RecA, (2) bound synthetic HJs with high affinity and structure specificity, (3) cleaved synthetic HJs into nicked DNA duplexes, and (4) cleaved the HJ by the introduction of symmetrically related nicks across the junction (Connolly and West 1990). The activ- 
H.D.M. Wyatt and S.C. West

A

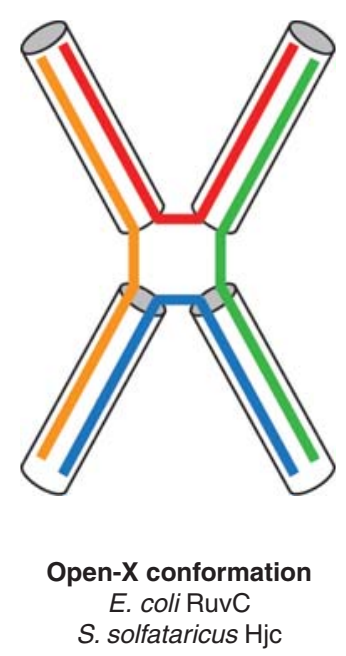

C

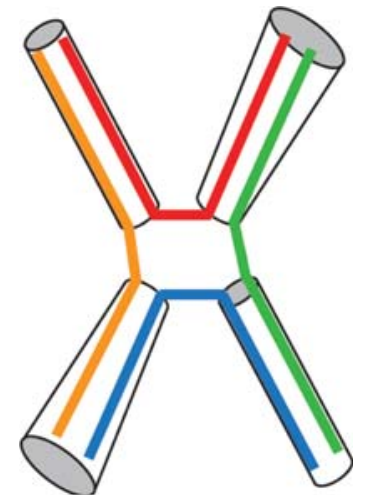

Distored open-X conformation T4 endonuclease VII

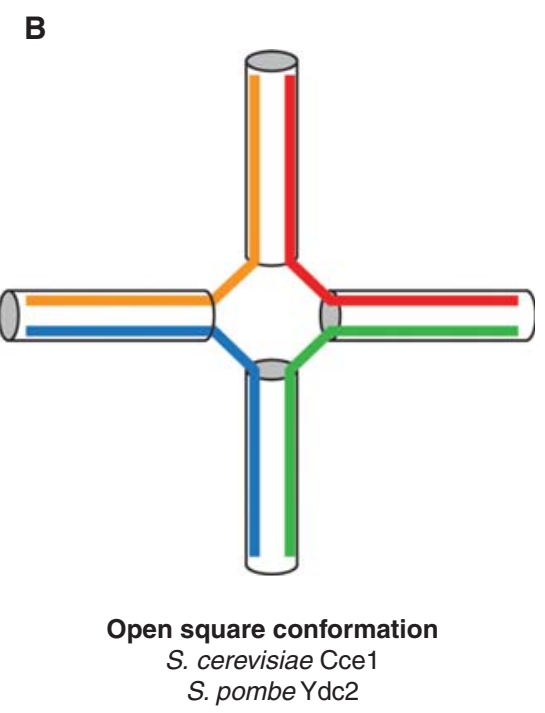

D

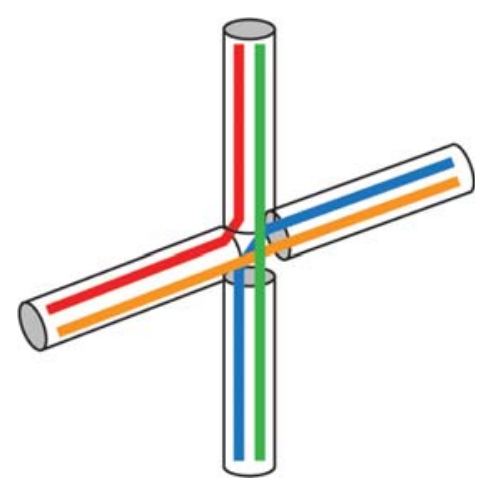

Coaxial conformation T7 endonuclease I

Figure 4. Global conformations of resolvase-bound Holliday junctions. Schematics showing the structural distortions that different canonical resolvases induce on binding the Holliday junction. Component helices are drawn in different colors according to the scheme used in Figure 2. (A) RuvC and Hjc induce an open-X conformation that retains twofold symmetry. $(B)$ Ccel and Ydc2 induce significant structural distortion to the junction to produce an open square conformation with fourfold symmetry. (C) T4 endonuclease VII manipulates the junction into a distorted open-X conformation. $(D)$ T7 endonuclease I induces a nearly perpendicular structure with the pairs of helical arms in a coaxial conformation. (Figure adapted from Declais and Lilley 2008.)

ity was subsequently identified as the product of the ruvC gene (Connolly et al. 1991; Dunderdale et al. 1991, 1994; Iwasaki et al. 1991; Sharples and Lloyd 1991).

The active form of RuvC is a homodimer containing two 19-kDa subunits (Dunderdale et al. 1991; Iwasaki et al. 1991). RuvC binds
DNA in a structure-specific manner such that HJs are bound with a $10^{3}$ - to $10^{4}$-fold higher affinity than duplex DNA (Bennett et al. 1993). The formation of RuvC-HJ complexes is independent of specific nucleotide sequences or metal ions (Dunderdale et al. 1991; Iwasaki et al. 1991; Bennett et al. 1993; Shah et al. 1994b; 
Takahagi et al. 1994). In contrast, divalent cations are required for DNA cleavage. In the presence of $\mathrm{Mg}^{2+}$ (or $\mathrm{Mn}^{2+}$ ), RuvC cleaves HJs by introducing symmetrically opposed nicks in strands of like polarity, at or near the junction (Dunderdale et al. 1991; Iwasaki et al. 1991; Bennett et al. 1993; Shah et al. 1994a; Takahagi et al. 1994). Importantly, the two incisions occur within the lifetime of a single protein-DNA complex (Fogg and Lilley 2000). Nonetheless, the two active sites can be uncoupled and therefore act sequentially during resolution (Shah et al. 1997; Fogg and Lilley 2000). To ensure that both strands of the junction are cleaved before the enzyme dissociates, the rate of second-strand cleavage is accelerated by several orders of magnitude compared to the first. The increased flexibility of a nicked HJ likely underpins this acceleration by promoting placement in the second active site for subsequent cleavage. The products of symmetrical $\mathrm{HJ}$ resolution are two nicked DNA duplexes with $5^{\prime}$-phosphate and $3^{\prime}$-hydroxyl termini that can be rejoined by DNA ligase (Bennett et al. 1993). Although RuvC binds $\mathrm{HJs}$ in a sequence-independent manner, the enzyme preferentially cleaves junctions containing the tetranucleotide consensus sequence $5^{\prime}{ }_{-} \mathrm{A} / \mathrm{T} T \downarrow^{\mathrm{C}>\mathrm{G}} / \mathrm{A}^{-3^{\prime}}$ (where $\downarrow$ indicates the site of incision) (Bennett et al. 1993; Shah et al. 1994a,b; Fogg et al. 1999). Optimal resolution occurs when the $5^{\prime}-\mathrm{TT} \downarrow-3^{\prime}$ incision site is positioned at, or one base pair away from, the point of strand exchange (Bennett and West 1996; Shida et al. 1996; Fogg et al. 1999; Sha et al. 2000). RuvC can also bind and cleave threestranded junctions, but it fails to act on Y substrates, mismatches, heteroduplex loops, and duplex DNA.

The crystal structure of E. coli RuvC shows that the two monomers are related by a dyad axis (Fig. 5A) (Ariyoshi et al. 1994; Ichiyanagi et al. 1998). The dimerization interface is formed mainly by two $\alpha / \beta$ helices that run parallel to the dyad axis. The most interesting structural feature of each subunit is a large cleft, with dimensions sufficient to accommodate duplex DNA. The walls of the cleft are lined with basic residues that mediate structure-specific DNA binding. The active site is located on the floor of the cleft and contains four acidic residues, namely, Asp-7, Glu-66, Asp-138, and Asp-141 (Ariyoshi et al. 1994; Saito et al. 1995; Ichiyanagi et al. 1998). This catalytic tetrad is expected to coordinate a pair of divalent metal cations and promote hydrolysis of the phosphodiester backbone (Saito et al. 1995; Yang et al. 2006). Importantly, the two DNA-binding clefts are separated by approximately $30 \AA$ (Ariyoshi et al. 1994). This is consistent with studies showing that RuvCholds the $\mathrm{HJ}$ in an unfolded conformation with twofold symmetry, such that base pairing is disrupted at the point of strand exchange (Fig. 4A) (Bennett and West 1995; Fogg et al. 2001). The surface between the two DNA-binding clefts contains an important aromatic reside, Phe-69, which stabilizes the open-X conformation and promotes DNA cleavage by forming a stacking interaction with a nucleotide base close to the branchpoint (Ariyoshi et al. 1994; Ichiyanagi et al. 1998; Yoshikawa et al. 2001).

Recently, the structures of Thermus thermophilus RuvC and a RuvC-HJ complex were solved (Fig. 5B) (Chen et al. 2013; Gorecka et al. 2013). The core consists of a five-stranded $\beta$-sheet, made from two parallel and three antiparallel strands, sandwiched between $\alpha$-helices-a characteristic feature of the retrovirus integrase superfamily (Nowotny 2009). As expected, the overall protein fold is similar to that of E. coli RuvC, but there are also some notable differences. First, the carboxyl termini of T. thermophilus RuvC is fully ordered, whereas E. coli RuvC contains a flexible carboxyl terminus. Moreover, the most carboxy-terminal residue of T. thermophilus RuvC (Leu-166) is engaged in intermolecular interactions between the two monomers. Second, the $\alpha$-helices of T. thermophilus RuvC are packed more tightly at the dimer interface. This region also contains more aromatic residues than are found in E. coli RuvC, such that T. thermophilus RuvC has a more tightly associated dimer interface, which may contribute to its natural thermostability.

In the unliganded structure, the two RuvC monomers are related by a pseudo twofold axis, with distinct asymmetry near the dimer interface (Chen et al. 2013). This region contains the amino terminus of $\alpha$-helix B from each subunit, 
H.D.M. Wyatt and S.C. West

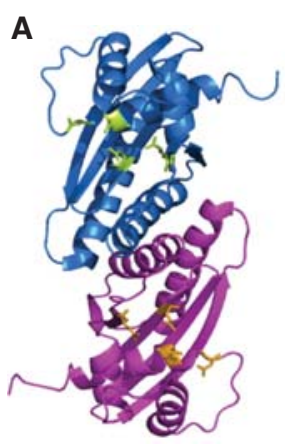

E. coli RuvC
B

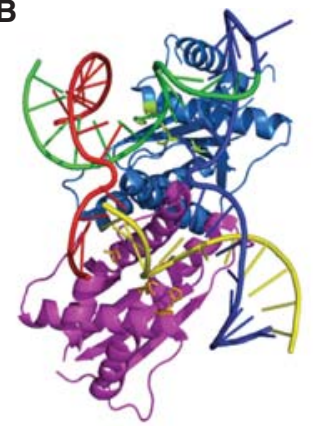

T. thermophilus RuvC-HJ complex
C

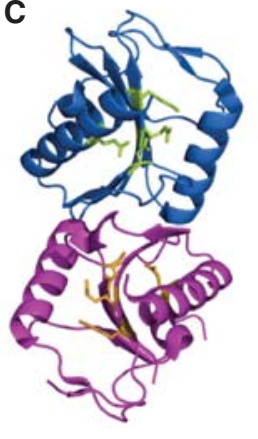

P. furiosus $\mathrm{Hjc}$

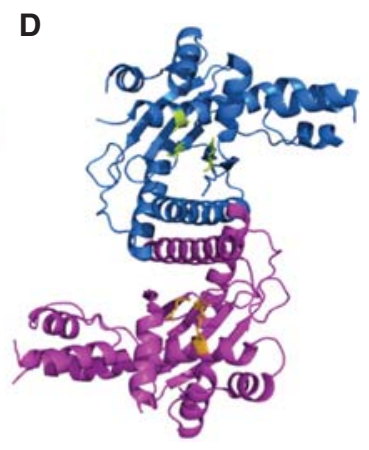

S. pombe Ydc2
E

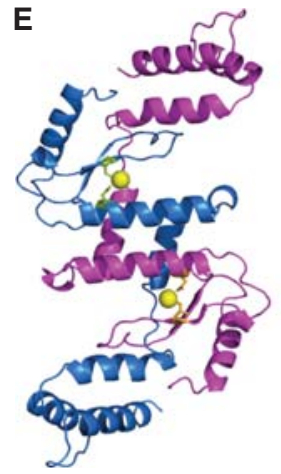

T4 endonuclease VII

G

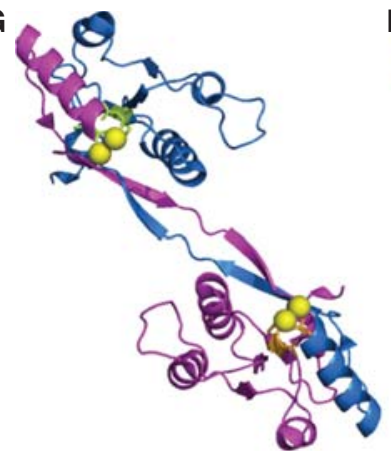

T7 endonuclease I
F

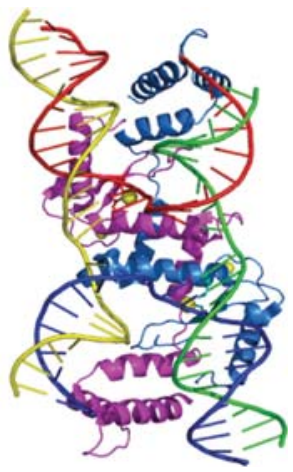

T4 endonuclease VII-HJ complex

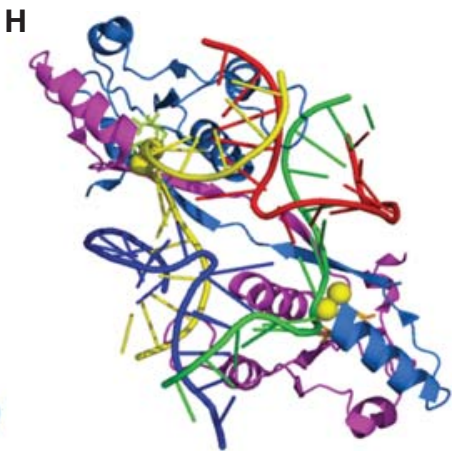

T7 endonuclease I-HJ complex

Figure 5. Crystal structures of canonical Holliday junction resolvases. For each structure, the view is approximately down the dyad axis of the protein and looking toward the predicted DNA-binding surface. Each structure depicts two monomeric subunits, one colored in blue and the other in magenta. Active site residues are illustrated in ball and stick format and colored in lime and orange. Metal ions bound in the active site are shown as yellow spheres. For RuvC $(A)$, T4 endonuclease VII $(F)$, and T7 endonuclease I $(G)$, the structures of the apoprotein and Holliday junction-bound enzyme are shown with the four DNA helices colored, as in Figure 2. The PDB IDs used to generate these structures are as follows: E. coli RuvC (A), 1hjr; T. thermophilus RuvC-HJ complex (B), 4ldo; P. furiosus Hjc $(C)$, 1gef; S. pombe Ydc2 (D), 1kcf; T4 endonuclease VII $(E)$, 1en7; T4 endonuclease VII-HJ complex $(F)$, 2qnc; T7 endonuclease I $(G), 1 \mathrm{~m} 0 \mathrm{~d}$; and T7 endonuclease I-HJ complex $(H), 2 \mathrm{pfj}$. 
the preceding loop, and catalytically important aromatic residues, such as Phe-73 (Phe-69 in E. coli RuvC), which adopts different conformations in the two monomers. Importantly, only one of the two active sites exists in a catalytically competent conformation. From a mechanistic perspective, this was initially interpreted as evidence that the two monomers undergo a "flipflop" motion, in which conformational changes promote the sequential strand cleavages of $\mathrm{HJ}$ resolution. However, in the structure of the RuvC-HJ complex, the loops from each monomer adopt a similar conformation, suggesting that more subtle differences in protein conformation or protein-DNA interactions may underpin the mechanism of $\mathrm{HJ}$ resolution (Gorecka et al. 2013). Comparison of the unliganded and DNA-bound structures reveals that the only major conformational change that occurs in RuvC on DNA binding is a reorganization of the loops that interact with the HJ substrate. Importantly, the RuvC-HJ crystal structure shows that the junction is bound in an unfolded, tetrahedral conformation with twofold symmetry (Fig. 5B). This tetrahedral geometry was also observed in biochemical studies of $E$. coli RuvC-HJ complexes (Fig. 4A) (Bennett and West 1995; Fogg et al. 2001), highlighting an evolutionarily conserved mechanism of DNA binding.

\section{The RuvABC Resolvasome}

Genetic and biochemical studies indicate that RuvAB-catalyzed branch migration is functionally coupled with RuvC-mediated $\mathrm{HJ}$ resolution (Sharples et al. 1990; Eggleston et al. 1997; Davies and West 1998; van Gool et al. 1998, 1999). It is thought that RuvA targets the resolvasome to the $\mathrm{HJ}$ and imposes an open configuration, such that RuvB can then use the energy of ATP hydrolysis to pump duplex DNA through the RuvABC complex. This provides an elegant mechanism for RuvC to scan the DNA for its preferred cleavage sequences. Once encountered, RuvC catalyzes $\mathrm{HJ}$ resolution and the RuvABC complex dissociates from DNA. However, despite the attractiveness of this model, it remains unclear how the RuvC dimer can dock onto the surface of the RuvA-HJ complex because of steric hindrance between the DNA backbone and symmetrical loops in the RuvC dimer. These constraints may be alleviated by the conformational changes that occur on DNA binding.

\section{HJ Resolvases in Archaea}

The first evidence for archaeal $\mathrm{HJ}$ resolvases came from biochemical studies showing that fractionated extracts of the euryarchaeote $P$. furiosus contained an activity that resolved HJs in vitro (Komori et al. 1999). The protein was subsequently purified and named Hjc, for Holliday junction cleavage. Two similar proteins have been identified in the crenarchaeote Sulfolobus solfataricus. One is the homolog of $P$. furiosus $\mathrm{Hjc}$ and the other is a related protein called Hje, for Holliday junction endonuclease (Kvaratskhelia and White 2000a,b). Although $\mathrm{Hjc}$ is conserved in all archaea, Hje has only been identified in $S$. solfataricus. As the open reading frame for $\mathrm{Hje}$ has not been identified, it is possible that Hje might represent a virally encoded protein (Middleton et al. 2004).

$\mathrm{Hjc}$ is a dimeric protein that binds preferentially to four-way DNA junctions with binding affinities in the low nanomolar range (Komori et al. 2000b; Kvaratskhelia and White 2000b). In the presence of $\mathrm{Mg}^{2+}, \mathrm{Hjc}$ catalyzes $\mathrm{HJ}$ resolution by introducing symmetrically opposed incisions across the branchpoint (Komori et al. 1999, 2000b; Kvaratskhelia and White 2000b). The major cleavage sites are located in the exchanging strands, positioned three bases on the $3^{\prime}$ side of the junction and are independent of nucleotide sequence. Importantly, the cleavage products can be joined by T4 DNA ligase, indicating that they contain symmetrically related nicks and 5'-phosphate and 3'-hydroxyl termini. In addition to cleaving four-way $\mathrm{HJ}$, low levels of Hjc-mediated cleavage are also observed on three-way junctions, but not linear DNA duplexes.

There are currently three different crystal structures of $\mathrm{Hjc}$; two represent $P$. furiosus $\mathrm{Hjc}$ and one represents $S$. solfataricus $\mathrm{Hjc}$ (Bond et al. 2001; Nishino et al. 2001a,b). Surprisingly, structure-function studies revealed that $\mathrm{Hjc}$ is 
remarkably similar to type II restriction endonucleases (Daiyasu et al. 2000; Komori et al. 2000a; Kvaratskhelia et al. 2000). The functional unit is a symmetric homodimer, in which residues from the $\beta$-sheets of each subunit form a central hydrophobic core that holds the two subunits together (Fig. 5C). The surface of the Hjc dimer contains several electropositive residues, most of which are clustered on one face and generate a large flat DNA-binding surface that traces around the conserved metal-binding sites in the shape of an "S." A flexible amino-terminal segment of $\mathrm{Hjc}$, composed of amino acids $1-5$, is crucial for stable protein-DNA contacts. The active site contains three acidic amino acids and one lysine residue, which form the conserved catalytic motifs of type II restriction endonucleases and are essential for Hjc activity. Notably, the two active sites in the $\mathrm{Hjc}$ dimer are separated by approximately $29 \AA$ (Bond et al. 2001; Nishino et al. 2001b). This spacing is consistent with biochemical and structural studies showing that $\mathrm{Hjc}$ manipulates the $\mathrm{HJ}$ into an open $\mathrm{X}$ shape with twofold symmetry (Fig. 4A) (Kvaratskhelia et al. 2000; Fogg et al. 2001). Hjc induces a local disruption of base pairing near the point of strand exchange, which is mediated by the flexible amino-terminal segment (Kvaratskhelia et al. 2000; Nishino et al. 2001a).

\section{Bacteriophage Resolvases}

Unlike cellular $\mathrm{HJ}$ resolvases that have specific roles in resolving four-way intermediates that form during DNA recombination and repair, the phage endonucleases have two additional and essential roles: (1) debranching DNA structures prior to packaging the viral genome into the head particles, and (2) degrading host DNA. As indicated in Table 1 and discussed below, this is reflected by the relatively broad substrate specificity displayed by the recombinant proteins in vitro.

\section{T4 Endonuclease VII}

The first biological evidence for a $\mathrm{HJ}$ resolvase activity came from studies of phage-infected bacteria. E. coli infected with bacteriophage T4 mutated in gene 49 were found to accumulate an aberrant form of replicating DNA that sediments much faster than that formed during wild-type infection (Frankel et al. 1971). This unusual form of DNA, termed very-fast sedimenting (VFS) DNA, is a network of highly branched recombination intermediates that cannot be packaged into phage heads. Mixing experiments with extracts from cells infected with gene $49^{+}$or gene $49^{-}$phage showed that VFS DNA could be converted to the normal form of DNA. Furthermore, the hydrolysis reaction depended on $\mathrm{Mg}^{2+}$ and was inhibited by excess DNA. Altogether, these results showed that the product of gene 49 was a phage-encoded endonuclease, which was subsequently named T4 endonuclease (endo) VII.

T4 endo VII is a dimeric protein that binds preferentially to four-way DNA junctions in a $\mathrm{Mg}^{2+}$ - and sequence-independent manner (Nishimoto et al. 1979; Parsons et al. 1990; Picksley et al. 1990; Giraud-Panis and Lilley 1996; Pöhler et al. 1996; Golz et al. 1997). Interactions between the amino and carboxyl termini of different monomers are essential for DNA binding (Birkenbihl and Kemper 1998). This ensures that only dimeric proteins interact with $\mathrm{HJ}$ substrates and thus guarantees that two active sites will be positioned on the junction for a coordinated resolution reaction. Biochemical studies revealed that T4 endo VII distorts the $\mathrm{HJ}$ into an open H-shaped structure with twofold symmetry (Fig. 4C) (Parsons et al. 1990; Pöhler et al. 1996). Importantly, the crystal structure of a catalytically inactive mutant bound to an immobile HJ shows that the enzyme protrudes into the center of the junction without disrupting the central base pairs (Fig. 5F) (Biertumpfel et al. 2007).

Consistent with important roles in DNA packaging and repair, T4 endo VII cleaves a multitude of DNA structures, including ssDNA, gapped DNA, heteroduplex loops, three-way junctions, Holliday junctions, apurinic/apyrimidinic lesions, and bulky adducts (Kemper and Garabett 1981; Mizuuchi et al. 1982; Kemper et al. 1984; Lilley and Kemper 1984; Jensch and Kemper 1986; Mueller et al. 1990; Picksley et al. 1990; Murchie and Lilley 1993; Greger and 
Kemper 1998). In each case, the enzyme introduces a pair of staggered nicks on either side of the structure to be removed. The nicks are always located on the $3^{\prime}$ side of the target DNA and occur in a sequential nick and counternick reaction. $\mathrm{HJ}$ resolution proceeds by a pair of nicks in strands of like polarity, each positioned one to five nucleotides on the $3^{\prime}$ side of the junction. T4 endo VII exhibits a bias toward cleaving the exchanging strands and on the $3^{\prime}$ side of thymine residues (Kemper et al. 1984; Duckett et al. 1988; Mueller et al. 1988; Picksley et al. 1990). The duplex products contain symmetrically related nicks with ligatable $5^{\prime}$-phosphate and $3^{\prime}$-hydroxyl termini (Mizuuchi et al. 1982; Lilley and Kemper 1984). Like RuvC, the two active sites of T4 endo VII can function independently (Giraud-Panis and Lilley 1997; Fogg and Lilley 2000), although the reaction involves two temporally coordinated cleavages that occur within the lifetime of the proteinDNA complex (Pottmeyer and Kemper 1992; Giraud-Panis and Lilley 1997).

The structures of T4 endo VII (Fig. 5E) and a T4 endo VII-HJ complex (Fig. 5F) have been determined (Raaijmakers et al. 1999, 2001; Biertumpfel et al. 2007), revealing that endo VII is a remarkably flexible protein with several unique features. First, the monomer represents a novel fold, composed almost entirely of $\alpha$ helices and containing two $\mathrm{C}-X-X-\mathrm{C}$ motifs that coordinate zinc binding. This contrasts other $\mathrm{HJ}$ resolvases, whose monomers contain stable $\alpha / \beta$ folds. Second, the dimer exhibits a unique domain-swapped architecture, in which the carboxyl terminus of one monomer interacts with the amino terminus of the other subunit. The two extensively intertwined monomers are related by a twofold symmetrical axis and form an elongated head-to-tail " $\mathrm{S}$ "-shaped dimer (Fig. 5E). Finally, the dimerization interface contains two pairs of antiparallel helices that cross each other at an angle of $70^{\circ}-80^{\circ}$, thus forming a novel "four-helix cross" motif, not found in any other $\mathrm{HJ}$ resolvase.

Similar to other $\mathrm{HJ}$ resolvases, the S-shaped dimer contains a pronounced bipolar charge distribution (Raaijmakers et al. 1999, 2001). The 3.1- $\AA$ resolution crystal structure of a T4 endo VII-HJ complex shows that the concave face (lined with solvent-exposed basic residues) is bound to the minor-groove side of the junction (Biertumpfel et al. 2007). The only direct interactions between T4 endo VII and DNA bases occur at the helical branchpoint. Here, residues from the "four-helix cross" motif penetrate into the junction, separating all four strands without any disruption in base pairing. The active sites, defined by a cluster of catalytically essential His and Asp residues, are positioned such that the exchanging strands will be cleaved two nucleotides from the branchpoint. Importantly, a comparison of the T4 endo VII crystal structures reveals a remarkable degree of conformational flexibility in the dimerization and DNA-binding domains (Raaijmakers et al. 1999, 2001). This likely underpins the enzyme's ability to recognize and cleave a broad range of branched DNA structures.

\section{T7 Endonuclease I}

T7 endo I (gp3) is required for the resolution of branched recombination intermediates and for the maturation and packaging of phage DNA (Tsujimoto and Ogawa 1978). The protein is dimeric and forms stable protein-DNA complexes (Parsons and West 1990; Picksley et al. 1990). Furthermore, catalytically inactive mutants bind synthetic HJs with high affinity, providing evidence that endonuclease activity and structure-selective DNA binding are separable processes (Duckett et al. 1995; Parkinson and Lilley 1997; Parkinson et al. 1999). The bound junction adopts a conformation that is quite different from that seen in other resolvase-HJ complexes. First, although coaxial helical stacking is maintained, there is a $90^{\circ}$ rotation of the axes, causing the arms to adopt a parallel, perpendicular orientation to each other (Fig. 4D) (Duckett et al. 1995; Declais et al. 2003). Second, the electrophoretic pattern of proteinDNA complexes provide evidence for the coexistence of T7 endo I in complex with both crossover isomers, with a 4:1 bias toward one of the two conformers. This is accompanied by a localized distortion of the base pairs at the point of strand exchange, which provides a strong 
contrast to the structure of the T4 endo VII-HJ complex. In the presence of $\mathrm{Mg}^{2+}$ or $\mathrm{Mn}^{2+}, \mathrm{T} 7$ endo I catalyzes the endonucleolytic cleavage of (1) secondary structures that form in ssDNA, (2) supercoiled plasmid DNA, and (3) various branched double-stranded DNA (ds DNA), substrates, including cruciforms, HJs, threeway junctions, and Y-shaped structures (Center and Richardson 1970; Sadowski 1971; de Massy et al. 1987; Müller et al. 1990; Picksley et al. 1990; Parkinson and Lilley 1997). T7 endo I shows a preference for cleavage between two pyrimidine residues (Picksley et al. 1990).

Crystallographic structures have been determined for T7 endo I and the T7 endo I-HJ complex (Hadden et al. 2001, 2002, 2007). The apo structure reveals a symmetric homodimer with two distinct catalytic domains separated by an elongated $\beta$-sheet bridge. As such, the overall architecture of $\mathrm{T} 7$ endo I resembles a curved barbell (Fig. 5G). As in T4 endo VII, the two monomers are extensively intertwined, to the extent that each domain is composed of elements from both subunits. An extensive dimeric interface accounts for this intimate association, which also explains the remarkable stability of the homodimer in free solution (Parkinson and Lilley 1997). T7 endo I also has a composite active site, in which each monomer contributes distinct catalytic residues (Declais et al. 2001; Hadden et al. 2001). The catalytic site contains a conserved P-D ... (D/E)-X-K motif found in several type II restriction enzymes and coordinates a two-metal ion mechanism of phosphodiester hydrolysis (Parkinson et al. 1999; Declais et al. 2001; Hadden et al. 2001, 2002).

The structure of T7 endo I-HJ complex (Fig. $5 \mathrm{H}$ ) shows that the protein straddles the HJ (by virtue of its electropositive surface) and wraps around it to a much greater extent than T4 endo VII (Fig. 5F) (Hadden et al. 2007). Residues in the amino and carboxyl termini are required for efficient DNA binding (Parkinson et al. 1999; Freeman et al. 2013), which triggers the formation of two long electropositive channels that are aligned nearly perpendicular to each other (Hadden et al. 2007). These channels are lined with basic residues that establish hydrogen bonds with the phosphate groups of the continuous strands, thus imparting T7 endo I with selectivity for DNA structures that can be modeled into this near-perpendicular geometry (Declais et al. 2006; Hadden et al. 2007). The two active sites are positioned such that the continuous strands will be cleaved one nucleotide on the $5^{\prime}$ side of the junction (Hadden et al. 2007). Importantly, the amino terminus facilitates strand separation at the junction center and thus, has an important role in mediating $\mathrm{HJ}$ resolution in vitro (Freeman et al. 2013).

\section{Junction-Resolving Enzymes in Eukaryotes}

The quest for eukaryotic HJ resolvases has been a slow and difficult task. The first evidence for a RuvC-like resolvase activity came from studies with yeast cell-free extracts (Symington and Kolodner 1985; West and Korner 1985). However, perhaps surprisingly, this activity turned out to be mitochondrial rather than nuclear (Kleff et al. 1992). Nonetheless, two distinct nuclear activities that cleaved HJs in vitro were identified in extracts prepared from animal tissues and human cells (Elborough and West 1990; Constantinou et al. 2002). The first activity was shown to cleave HJs in a manner similar to RuvC (i.e., canonical resolution). The second activity, which contained the MUS81 protein, exhibited a noncanonical mechanism of resolution. Unfortunately, because of its low abundance, the gene encoding the canonical resolvase remained elusive for several years. In the following section, we will first discuss the yeast mitochondrial HJ resolvases Cce1 and Ydc2. This will be followed by a description of three mammalian nuclear enzymes (and their orthologs in yeast, flies, and worms) that have been implicated in HJ resolution, namely, GEN1, MUS81-EME1, and SLX1-SLX4.

\section{Mitochondrial HJ Resolvases: Yeast Cce1 and Ydc2}

The HJ resolvase activity present in S. cerevisiae extracts was identified as the product of the CCE1 (for cruciform cutting endonuclease) gene (Kleff et al. 1992), but, surprisingly, CCE1 
null strains exhibit no obvious growth defects or defects in recombination. However, the mutant phenotypes suggest abnormal mitochondrial function: (1) an elevated frequency of petite cells, (2) cytoplasmic aggregation of mitochondrial DNA (mtDNA), and (3) increased mitotic loss of mtDNA. Deletion of CCE1 also results in the accumulation of branched mtDNA structures, which form large networks that are interlinked by unresolved three- and four-way junctions (Lockshon et al. 1995). These observations are consistent with data showing that Ccel is localized exclusively in the mitochondria and that CCE1 is allelic to MGT1, which is essential for mitochondrial genome transmission (Ezekiel and Zassenhaus 1993). The S. pombe homolog of Cce1 is Ydc2 (Whitby and Dixon 1997; White and Lilley 1997a; Oram et al. 1998). Similar to yeast lacking CCE1, Ydc2-deficient cells contain large aggregates of mtDNA and fewer mtDNA nucleoids than wild-type cells (Doe et al. 2000).

Similar to other $\mathrm{HJ}$ resolvases, Ccel and Ydc2 have a high binding affinity for four-way DNA junctions (White and Lilley 1996, 1997a; Whitby and Dixon 1997; Oram et al. 1998). In addition, both proteins bind to HJs as dimers (White and Lilley 1996, 1997b). Substrate binding is mediated by structural selectivity and occurs independently of nucleotide sequence and divalent metal cations (White and Lilley 1996, 1997b; Schofield et al. 1998). Cce1 and Ydc2 both manipulate the HJ into a fully extended, open square structure, in which the four central bases are unpaired (Fig. 4B) (Declais and Lilley 2000). This structure is remarkably similar to that of the protein-free $\mathrm{HJ}$ in the absence of metal ions (Fig. 2A) and emphasizes the conformational flexibility of $\mathrm{HJ}$ resolvases and their DNA substrates.

Although Cce1/Ydc2 bind DNA substrates in the absence of metal ions, DNA cleavage requires $\mathrm{Mg}^{2+}$ or $\mathrm{Mn}^{2+}$. The nucleases are active on HJs, whereas weak or no endonuclease activity is observed with three-way junctions in vitro. Cleavage occurs by the introduction of two sequential nicks across the junction within the lifetime of the protein-DNA complex, with a large rate enhancement for second-strand cleavage (Fogg et al. 2000). This ensures that bilateral cleavage occurs in a tightly coordinated manner, such that HJs are efficiently resolved into nicked DNA duplexes that can be ligated. Ccel exhibits a strong preference for cleaving HJs with the consensus sequence $5^{\prime}-\mathrm{ACT}^{\downarrow} \mathrm{A}-3^{\prime}$ (White and Lilley 1996; Schofield et al. 1998), whereas Ydc2 cleaves $5^{\prime}-\mathrm{C} / \mathrm{T}^{\downarrow}-3^{\prime}$ (Whitby and Dixon 1997; White and Lilley 1997a; Oram et al. 1998). Optimal activity for both enzymes is observed when the target sequence is located in the continuous strands and at the point of strand exchange. Collectively, these studies indicate that Ccel and Ydc2 represent canonical HJ resolvases that are remarkably similar to RuvC. Additional support for this conclusion comes from the finding that Ydc2 overexpression in resolvase-deficient $E$. coli is sufficient to complement the UV sensitivity of these cells (Whitby and Dixon 1997).

The crystal structure of Ydc2 reveals a dimeric protein with an overall "S" shape (Fig. $5 \mathrm{D})$, the core of which is structurally homologous to RuvC (Ceschini et al. 2001). The Sshaped structure is remarkably similar to that of T4 endo VII, despite the fact that the two proteins are unrelated in sequence and fold (Raaijmakers et al. 1999; Ceschini et al. 2001). Similar to other $\mathrm{HJ}$ resolvases, hydrophobic side chains from each Ydc2 monomer contribute to the dimeric interface. In addition, the Ydc2 dimer presents two distinct faces, one of which is flattened and enriched in basic residues that mediate protein-DNA interactions (Ceschini et al. 2001; Sigala and Tsaneva 2003). One interesting structural feature is the presence of a small $\alpha$-helical bundle (triple-helix domain) that has no equivalent in RuvC. The triple-helix domain, located in the electropositive face, protrudes outward and is important for substrate recognition and cleavage. A conserved patch of acidic residues is located near the "elbow" between the laterally protruding triple-helix domain and the central dimerization interface, and these residues form the catalytic triad that is essential for enzyme activity. The two active sites in the Ydc2 dimer are separated by approximately $34 \AA$ (Ceschini et al. 2001), in good agreement with the finding that Ydc2 unfolds 
the $\mathrm{HJ}$ into an open square-planar conformation in which the scissile bonds in the continuous strands are separated by $30-35 \AA$ (Roe et al. 1998; White and Lilley 1998). Another intriguing feature in Ydc2 is the occurrence of small flexible loops in the DNA-binding region (Ceschini et al. 2001). One of these loops extrudes from the surface to form a pin structure that is positioned near the dimer interface. In the modeled Ydc2-HJ complex, the pin fits neatly into the center of the open junction and may contribute to sequence specificity and/or base pair disruption. Moreover, the pin is thought to act as a conformational sensor that relays active site conformation to the dimeric interface, which promotes optimal positioning for metal ion binding and catalysis.

\section{Canonical Nuclear HJ Resolvases: GEN1/Yen1}

The first evidence for a canonical mammalian $\mathrm{HJ}$ resolvase was the detection of a RuvC-like nuclease activity in fractionated cell-free extracts prepared from homogenized calf-thymus tissue (Elborough and West 1990). The activity was subsequently observed in nuclear extracts from tissue-cultured human cells (Hyde et al. 1994; Constantinou et al. 2001), and the gene product was eventually identified as GEN1 (Ip et al. 2008). The human protein, GEN1, and its $S$. cerevisiae ortholog, Yen1, represent a subclass of the Rad2/XPG family of endonucleases (Lieber 1997). GEN1 orthologs are present in higher eukaryotes, including the rice Oryza sativa (OsSEND-1), Caenorhabditis elegans (GEN1), and Drosophila melanogaster (DmGEN), but are absent from S. pombe (Furukawa et al. 2003; Ishikawa et al. 2004; Ip et al. 2008). The Rad2/ XPG family includes several well-characterized nucleases, such as the nucleotide excision repair protein XPG and the $5^{\prime}$-flap endonuclease FEN1. These proteins are defined by the presence of three characteristic motifs: (1) an XPGtype amino-terminal domain (XPG-N), (2) a conserved nuclease domain positioned in the central region of the protein (XPG-I), and (3) a helix-hairpin-helix domain that flanks the nuclease domain.
Studies of an amino-terminal fragment of human GEN1 (GEN1 ${ }^{1-527}$ ), containing the nuclease active site, revealed $\mathrm{Mg}^{2+}$-dependent incision of $5^{\prime}$ flaps, replication forks, and nicked or intact HJ structures (Ip et al. 2008; Rass et al. 2010). In contrast, no activity is observed with $3^{\prime}$ flaps, splayed arms, duplex DNA, or single-stranded substrates. Similar to other canonical $\mathrm{HJ}$ resolvases, GEN1 cleaves HJs into nicked duplex products by introducing a pair of symmetrically opposed incisions across the junction to generate ligatable products. GEN1 cleaves immobile HJs at a unique site, positioned one nucleotide on the $3^{\prime}$ side of the branchpoint. In addition, GEN1 preferentially cleaves the continuous strands of the HJ. At the present time, the sequence specificity has not been determined. One distinguishing feature of GEN1 $1^{1-527}$, although this has yet to be shown for the full-length protein, is that it is monomeric in solution, whereas other resolvases are stable dimers (Table 1). Nonetheless, GEN $1^{1-527}$ dimerizes on the $\mathrm{HJ}$ substrate to ensure that twin active sites are positioned on the junction for coordinated resolution.

The ability of human GEN1 to resolve HJs in vivo is supported by genetic data showing that ectopic expression of human GEN1 ${ }^{1-527}$ promotes $\mathrm{HJ}$ resolution and $\mathrm{CO}$ formation in HR-deficient $S$. pombe mus $81 \Delta$ strains (Lorenz et al. 2010). In addition, ectopically expressed GEN $1^{1-527}$ resolves recombination intermediates that otherwise accumulate in methyl methanesulfonate (MMS)-treated S. cerevisiae sgs1 or top3 mutants (Mankouri et al. 2011). Moreover, microinjection of human GEN1 $1^{1-527}$ into the germline of C. elegans mus-81 xpf-1 mutants eliminates persistent DNA bridges (i.e., unresolved HJs) that form between bivalent chromosomes (O'Neil et al. 2013). In mitotic human cells, transient small interfering RNA (siRNA)mediated depletion of GEN1 disrupts mitotic progression and increases the frequency of multi- and micronucleated cells, centrosomal defects, DNA bridges, and aberrant chromosome formation (Gao et al. 2012; Rodrigue et al. 2013). These phenotypes indicate that human GEN1 is important for the removal of DNA structures that impede chromosome seg- 
regation and provide further evidence that GEN1 functions as a canonical $\mathrm{HJ}$ resolvase. However, it is noteworthy that human cells depleted of GEN1 display little sensitivity to DNAdamaging agents such as cisplatin, camptothecin (CPT), or MMS (Svendsen et al. 2009; Wyatt et al. 2013). Furthermore, yen $1 \Delta$ budding yeast are resistant to genotoxic agents (e.g., 4-nitroquinoline-1-oxide [4NQO], hydroxyurea [HU], MMS) and exhibit wild-type levels of cell growth, viability, and meiotic CO formation (Blanco et al. 2010; Ho et al. 2010; Matos et al. 2011; de Muyt et al. 2012; Zakharyevich et al. 2012). These results can be explained by the presence of nuclear enzymes that (1) act redundantly with GEN1/Yen1, and/or (2) compete for the same or similar substrates and thus mask the phenotype associated with the loss of GEN1/Yen1 (e.g., the BLM/Sgs1 helicase and other structure-selective endonucleases).

Recent genetic studies in yeast have uncovered a complex relationship between Yen1 and two noncanonical HJ resolvases, namely, Mus81-Mms4 and Slx1-Slx4 (Blanco et al. 2010; Ho et al. 2010; Matos et al. 2011, 2013; de Muyt et al. 2012; Gallo-Fernandez et al. 2012; Zakharyevich et al. 2012; Szakal and Branzei 2013). Specifically, Yen 1 activity is only required in cells lacking Mus81-Mms4, suggesting that it provides a backup function for Mus81-Mms4. The observation that yen $1 \Delta$ mus $81 \Delta$ double mutants exhibit a synthetic phenotype indicates that the enzymes define two distinct pathways of HJ resolution (Blanco et al. 2010; Ho et al. 2010; Matos et al. 2011, 2013). Synthetic lethality has also been observed between $D$. melanogaster Gen and Mus81, in which double mutants exhibit significantly increased levels of apoptosis (Andersen et al. 2011). In contrast to yeast, however, flies rely predominantly on $D m G E N$ for the resolution of DNA repair and/or recombination intermediates (Andersen et al. 2011). In C. elegans, the loss of gen-1 enhances the severity of the mus- 81 phentyope, in terms of embryonic lethality, brood size, and meiotic chromosomal abnormalities (O'Neil et al. 2013; Saito et al. 2013). Moreover, the synthetic phenotype of gen-1 sl $x-1$ mutants reveals that GEN-1 and SLX-1 define two separate pathways for process- ing recombination intermediates in vivo (Saito et al. 2012, 2013). A similar relationship exists in mitotic human cells in which transient depletion of GEN1 and either MUS81, SLX1, or SLX4 generates an additive phenotype in terms of SCE frequency, chromosome abnormalities, and cell death (Wechsler et al. 2011; Garner et al. 2013; Wyatt et al. 2013; Sarbajna et al. 2014). Notably, the phenotypes associated with the loss of GEN1 and either MUS81, SLX1, or SLX4 are particularly severe in the absence of the BLM helicase. This observation emphasizes that both $\mathrm{HJ}$ dissolution and $\mathrm{HJ}$ resolution (Fig. 1) are required for the efficient removal of DNA structures that would otherwise impede normal cell cycle progression.

\section{Noncanonical Nuclear HJ Resolvases: MUS81-} EME1/Mus81-Mms4

The Mus81 protein was initially discovered through its physical association with the $S$. pombe replication checkpoint kinase Cds1 (Boddy et al. 2000) and the S. cerevisiae recombination/repair protein RAD54 (Interthal and Heyer 2000), as well as in a synthetic-lethal screen for genes required in the absence of the Sgs1 helicase (Mullen et al. 2001). MUS81/ Mus81 is a member of the ERCC4/XPF family of structure-selective endonucleases, which also includes the XPF-ERCC1 nucleotide excision repair endonuclease (Ciccia et al. 2008). MUS81/Mus81 contains tandem helix-hairpin-helix motifs in the carboxyl terminus, which likely mediate DNA binding, as well as a conserved endonuclease domain in the central region of the protein, which contains the catalytic G-D-X ${ }_{n}-\mathrm{E}-\mathrm{R}-\mathrm{K}-\mathrm{X}_{3}-\mathrm{D}$ motif (Nishino et al. 2003; Chang et al. 2008). Similar to other members of the ERCC4/XPF family, MUS81/Mus81 forms a stable heterodimer with a noncatalytic subunit called EME1 in humans (Ciccia et al. 2003; Ögrünc and Sancar 2003), Mms4 in budding yeast (Kaliraman et al. 2001; Mullen et al. 2001), and Emel in fission yeast (Boddy et al. 2001). The formation of a functional heterodimer is supported by the findings that yeast mus81 and mms4/eme1 double mutants have identical phenotypes (Boddy et al. 2001; de los 
Santos et al. 2001; Kaliraman et al. 2001; Mullen et al. 2001; Doe et al. 2002).

Genetic studies revealed that Mus81-Mms4 has important roles in (1) cellular resistance to genotoxic agents (e.g., MMS and UV), (2) resolution of potentially toxic meiotic and mitotic recombination intermediates, and (3) maintenance of genomic stability (Boddy et al. 2000, 2001; Interthal and Heyer 2000; de los Santos et al. 2001; Kaliraman et al. 2001; Mullen et al. 2001; Doe et al. 2002; Smith et al. 2003; Ho et al. 2010; Dayani et al. 2011). In S. pombe mus81 mutants, ectopic expression of RusA (a cryptic bacteriophage $\mathrm{HJ}$ resolvase) restores cellular resistance to genotoxic agents and productive meiosis (Boddy et al. 2001; Doe et al. 2002). In contrast to fungi, loss of MUS81-EME1 has little, if any, impact on meiotic progression in mice (Abraham et al. 2003; McPherson et al. 2004; Dendouga et al. 2005), flies (Johnson-Schlitz and Engels 2006; Trowbridge et al. 2007), or worms (Agostinho et al. 2013; O'Neil et al. 2013; Saito et al. 2013). Nonetheless, these mutants exhibit mitotic defects and are hypersensitive to specific DNA-damaging agents, including CPT, cisplatin, and MMC. The observation that MUS81 is down-regulated in hepatocellular and colon carcinoma provides circumstantial evidence that MUS81-EME1 is also required for chromosomal stability in humans ( $\mathrm{Wu}$ et al. 2008, 2011).

Importantly, in yeast lacking the Sgs $1 /$ Rqh 1 helicase, Mus81-Mms4/Eme1 is essential for cell viability and the resolution of aberrant recombination intermediates in meiosis (Boddy et al. 2001; Kaliraman et al. 2001; Mullen et al. 2001; Doe et al. 2002; Jessop and Lichten 2008; Oh et al. 2008). A similar relationship exists in higher eukaryotes because C. elegans mus-81 him-6 and D. melanogaster mus 81 mus 309 mutants exhibit severe defects in mitosis and meiosis (HIM-6 and MUS-309 are orthologs of the BLM/Sgs1 helicase) (Johnson-Schlitz and Engels 2006; Trowbridge et al. 2007; Agostinho et al. 2013; O'Neil et al. 2013). Moreover, BLMdeficient mammalian cells require MUS81 for cell viability and SCE formation (Wechsler et al. 2011; Castor et al. 2013; Wyatt et al. 2013). Collectively, these observations indicate that (1)
MUS81-EME1/Mms4 is involved in the processing of mitotic and meiotic recombination intermediates, and (2) HJ dissolution and resolution are both required for the efficient removal of potentially toxic recombination intermediates.

Initial in vitro studies with partially purified MUS81-EME1 from human cells (Chen et al. 2001; Constantinou et al. 2002) or fission yeast (Boddy et al. 2001) showed $\mathrm{Mg}^{2+}$-dependent cleavage of synthetic HJs in a manner that was significantly different from that of RuvC. Specifically, HJs are resolved with asymmetric nicks, thus generating one gapped and one flapped DNA duplex that cannot be ligated in vitro (Boddy et al. 2001; Chen et al. 2001; Constantinou et al. 2002). Moreover, in contrast to canonical HJ resolvases, purified MUS81EME1/Mms4 cleaves intact HJs with very low efficiency compared to nicked HJs, $3^{\prime}$ flaps, and replication forks (Kaliraman et al. 2001; Ciccia et al. 2003; Gaillard et al. 2003; Fricke et al. 2005; Ehmsen and Heyer 2008; Taylor and McGowan 2008; Wyatt et al. 2013). This is consistent with observations showing that MUS81-EME1/ Mms4 is a single heterodimer in solution or when bound to DNA (Chang et al. 2008; Schwartz et al. 2012). However, these biochemical observations appear to contradict the genetic data showing that MUS81-EME1/Mms4 is important for $\mathrm{HJ}$ resolution and, indeed, have sparked a long-standing controversy regarding the role of MUS81-EME1/Mms4 in HJ resolution (Haber and Heyer 2001).

Two hypotheses have been proposed to reconcile the apparent discrepancies surrounding the activity of MUS81-EME1/Mms4 on HJs, and these are not necessarily mutually exclusive scenarios. First, nicked HJs may be the true substrates for MUS81-EME1/Mms4; the first nick arises from the initial strand invasion event that precedes the formation of a joint molecule. Alternatively, it might be introduced by another endonuclease, thus creating a substrate that could be "counternicked" by MUS81-EME1/ Mms4. Second, the MUS81-EME1/Mms4 enzyme itself might need to be "activated" to resolve intact HJs, and this could involve a posttranslational modification and/or interaction 
with another protein. Support for the second hypothesis comes from recent studies showing that Mus81-Mms4 is phosphorylated and activated at $\mathrm{G}_{2} / \mathrm{M}$ by the actions of $\mathrm{Cdk}$ and the polo-like kinase Cdc5 (Matos et al. 2011, 2013; Gallo-Fernandez et al. 2012; Szakal and Branzei 2013). Thus, the nuclease activity of Mus81Mms4 is temporally regulated to ensure that meiotic and mitotic recombination intermediates are eliminated prior to chromosome segregation, while also limiting the possibility of replication fork cleavage that could drive genomic instability. Interestingly, however, phosphorylation of Mus81-Mms4 does not induce dimerization, and, therefore, the mechanistic details of its activation remain unknown (Schwartz et al. 2012).

In human cells, the mechanism of activation of MUS81-EME1 also involves CDK and the polo kinase PLK1, but, in this case, phosphorylation leads to interactions with another structure-selective endonuclease implicated in $\mathrm{HJ}$ resolution, namely, SLX1-SLX4 (Andersen et al. 2009; Fekairi et al. 2009; Munoz et al. 2009; Svendsen et al. 2009; Wyatt et al. 2013). Biochemical and/or genetic interactions between MUS81-EME1 and SLX1-SLX4 have also been reported in mouse (Castor et al. 2013) and C. elegans (Agostinho et al. 2013; Saito et al. 2013). These observations, taken together with the finding that MUS81 and SLX1-SLX4 are epistatic in terms of SCE formation (Wechsler et al. 2011; Castor et al. 2013; Garner et al. 2013; Wyatt et al. 2013), indicate that cell-cycleregulated phosphorylation of MUS81-EME1 triggers its interaction with SLX1-SLX4 and that these enzymes cooperate to resolve intact HJs.

\section{Noncanonical Nuclear HJ Resolvases: SLX1-SLX4/SIX1-SIX4}

The SLX1 and SLX4 genes were first identified in $S$. cerevisiae in a synthetic-lethal screen for proteins that are required for viability in the absence of SGS1(Mullen et al. 2001). Likewise, $S$. pombe Slx 1 and Slx4 are essential in cells lacking the Rqh1 helicase (Coulon et al. 2004). A similar synthetic relationship exists between these genes in flies (Andersen et al. 2009,
2011) and worms (Agostinho et al. 2013; Saito et al. 2013). The mammalian SLX1 and SLX4 proteins were identified in 2009 (Fekairi et al. 2009; Munoz et al. 2009; Svendsen et al. 2009). In all organisms studied to date, SLX1 and SLX4 interact to form a stable heterodimer, although the formation of higher-order oligomers has not yet been excluded. Sequence alignment reveals that SLX1 contains a conserved UvrC-intron (URI)-endonuclease domain in the amino terminus and a carboxy-terminal RING/PHDtype zinc finger domain and, thus, belongs to the GIY-YIG superfamily of endonucleases (Dunin-Horkawicz et al. 2006). In contrast, the amino acid sequence of SLX4 is evolutionarily diverse. Nonetheless, fungal and human SLX4 proteins share a conserved carboxy-terminal SAP domain that is thought to mediate DNA binding and substrate specificity. Human SLX4 is a large, multidomain protein that provides a scaffold for the binding of several other proteins, including the MUS81-EME1 and XPF-ERCC1 nucleases (Andersen et al. 2009; Fekairi et al. 2009; Munoz et al. 2009; Svendsen et al. 2009).

Full-length yeast Slx1-Slx4 and human SLX1-SLX4 have been biochemically purified and are versatile $5^{\prime}$ flap endonucleases that cleave a variety of branched DNA structures, including $5^{\prime}$ and $3^{\prime}$ flaps, splayed arms, replication forks, stem loops, and synthetic HJs containing a mobile or immobile core (Fricke and Brill 2003; Coulon et al. 2004; Wyatt et al. 2013). Negligible cleavage is observed with ssDNA, dsDNA, or DNA duplexes containing either a nick or single-stranded extension. Endonuclease activity requires the presence of divalent cations $\left(\mathrm{Mg}^{2+}\right.$ or $\left.\mathrm{Mn}^{2+}\right)$ and is abolished by $\mathrm{mu}-$ tation of conserved catalytic residues in SLX1. The ability of SLX1-SLX4/Slx1-Slx4 to cleave diverse DNA structures is reminiscent of the broad substrate specificity displayed by bacteriophage resolvases such as T4 endo VII and T7 endo I. However, whereas the bacteriophage resolvases cut HJs by the introduction of a pair of coordinated and symmetric nicks, the SLX proteins promote asymmetric nicking to generate duplex products that are poorly ligatable. Mechanistic studies of human SLX1-SLX4 show that the nuclease introduces a nick near 
H.D.M. Wyatt and S.C. West

the base of the $\mathrm{HJ}$ and then dissociates before the second nick can occur, thus deviating from the classical RuvC-like mechanism of $\mathrm{HJ}$ resolution (Fig. 3) (Wyatt et al. 2013).

In yeast, slx 1 and $s l x 4$ single mutants exhibit wild-type growth rates, chromosome segregation, and sporulation efficiency (Mullen et al. 2001). However, the loss of either protein results in sensitivity to MMS (Fricke and Brill 2003) and elevated levels of spontaneous genome rearrangements (Zhang et al. 2006). Moreover, both genes exhibit synthetic lethality with sgs1/rqh1 mutants (Mullen et al. 2001; Coulon et al. 2004) and this is not suppressed in a rad52 background (Fricke and Brill 2003). This provides an interesting contrast to the observation that the synthetic lethality of $s g s 1$ and mus 81 or mms 4 mutants is rescued by mutations in HR genes, including RAD52 (Fabre et al. 2002; Bastin-Shanower et al. 2003). Collectively, these results suggest that yeast Mus81-Mms4 and Slx1Slx4 act on different DNA structures in vivo, which is consistent with the promiscuous nuclease activities of Slx1-Slx4 in vitro (Fricke and Brill 2003; Coulon et al. 2004). More specifically, in the absence of Sgs1/Rqh1, Mus81Mms4 is important for the resolution of toxic recombination intermediates, whereas Slx1 and Slx4 are required to maintain the structural integrity of replicating ribosomal DNA (rDNA) presumably by resolving recombination intermediates that are generated at stalled replication forks (Kaliraman and Brill 2002; Coulon et al. 2004, 2006).

Genetic studies in worms and flies indicate that SLX1 and/or SLX4 have important roles in processing recombination intermediates that arise at stalled or collapsed replication forks in both mitotic and meiotic cells, particularly in the absence of the BLM helicase (Andersen et al. 2009, 2011; Saito et al. 2012; Agostinho et al. 2013). In mouse, disruption of Giyd2/Slx1 or Btbd12/Slx4 results in a hypersensitivity to DNA-damaging agents that cause DNA interstrand cross-links (ICLs), which require recombination for repair (Crossan et al. 2011; Castor et al. 2013). However, $S l x 1^{-/-}$mice are born at Mendelian frequencies, are fertile, and do not exhibit any overt morphological, developmen- tal, or hematological defects (Castor et al. 2013). In contrast, $S l \times 4^{+/-}$and $S l \times 4^{-/-}$mice are born at sub-Mendelian ratios and display growth retardation and reduced fertility owing to altered meiotic progression and genomic instability (Crossan et al. 2011; Holloway et al. 2011; Castor et al. 2013). The phenotypic differences between Slx1- and Slx4-compromised animals has been attributed to (1) redundancy between SLX1 and other structure-selective nucleases, and (2) the observation that SLX4 interacts with multiple DNA-repair proteins in higher eukaryotes.

In somatic human cells, transient depletion of SLX1 or SLX4 causes sensitivity to camptothecin and genotoxic agents that cause ICLs (Andersen et al. 2009; Fekairi et al. 2009; Munoz et al. 2009; Svendsen et al. 2009). Importantly, biallelic mutations of the SLX 4 gene have been identified in patients with Fanconi anemia, a rare genetic disorder characterized by hypersensitivity to ICL agents, bone marrow failure, genome instability, and cancer predisposition (Kim et al. 2011; Stoepker et al. 2011). Also, the defects observed when mammalian SLX1SLX4 function is compromised are exacerbated in the absence of BLM or in the presence of exogenous DNA damage (Castor et al. 2013; Garner et al. 2013; Wyatt et al. 2013).

\section{SLX-MUS: A Unique HJ Resolvase in Higher Eukaryotes}

It is now clear that the $\mathrm{HJ}$ resolvases are involved in recombination to different extents, depending on the organism, cell type (mitotic or meiotic), and whether recombination is induced by naturally occurring events or exposure to specific genotoxic agents. In all cases, however, the resolvases are largely dispensable in cells containing BLM/Sgs (i.e., HJ dissolution). This observation has led to the notion that $\mathrm{HJ}$ resolution may provide a ubiquitous back-up mechanism for $\mathrm{HJ}$ dissolution, and together, these pathways ensure that HJs are efficiently removed prior to cell division. In mitotic human cells, it is now clear that there are at least two distinct pathways of $\mathrm{HJ}$ resolution: one mediated by MUS81-EME1 and SLX1-SLX4 
(termed SLX-MUS), and the other mediated by GEN1 (Fig. 6) (Wechsler et al. 2011; Castor et al. 2013; Garner et al. 2013; Wyatt et al. 2013). Specifically, MUS81, SLX1, SLX4, and GEN1 are required (to different extents) for cell viability, chromosome segregation, and for the elevated levels of SCEs that occur in BLM-deficient cells. Moreover, MUS81 and SLX1-SLX4 (but not GEN1) are epistatic with respect to SCE frequency (i.e., HJ processing) and chromosome stability in BLM-deficient cells.

Consistent with the epistatic relationship between MUS81-EME1 and SLX1-SLX4, physical interactions between the human proteins have been observed at the onset of mitosis, which correlates with MUS81-EME1 and SLX4 phosphorylation (Wyatt et al. 2013). Further- more, coexpression of the human MUS81EME1 and SLX1-SLX4 proteins in insect cells is sufficient for their direct interaction, providing evidence for a stable SLX-MUS enzyme that is amenable to biochemical purification. Moreover, SLX-MUS can be reconstituted in vitro using purified full-length MUS81-EME1 and SLX1-SLX4 proteins (Wyatt et al. 2013). These studies showed that SLX-MUS is more efficient at cleaving synthetic HJ substrates than either of the two component nucleases. Furthermore, $\mathrm{HJ}$ resolution proceeds by a coordinated nick and counternick mechanism, within the lifetime of the protein-DNA complex. Mixing experiments performed with catalytically inactive SLX1 ${ }^{\text {E82A }}-$ SLX4 and MUS81 ${ }^{\text {D307A }}$-EME1 mutants revealed that SLX1 catalyzes the initial,

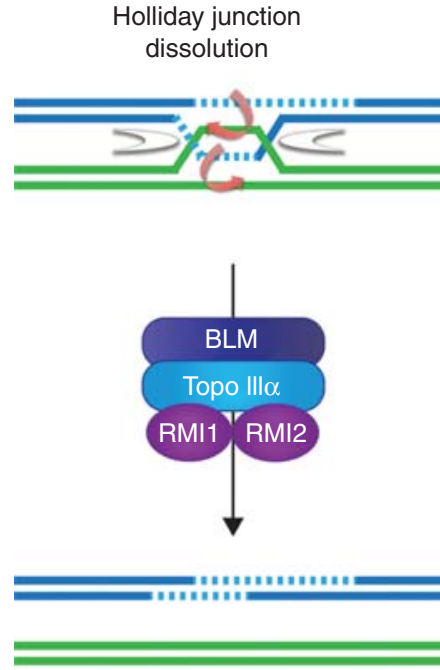

Noncrossover products Limits sister-chromatid exchanges Avoids loss of heterozygosity
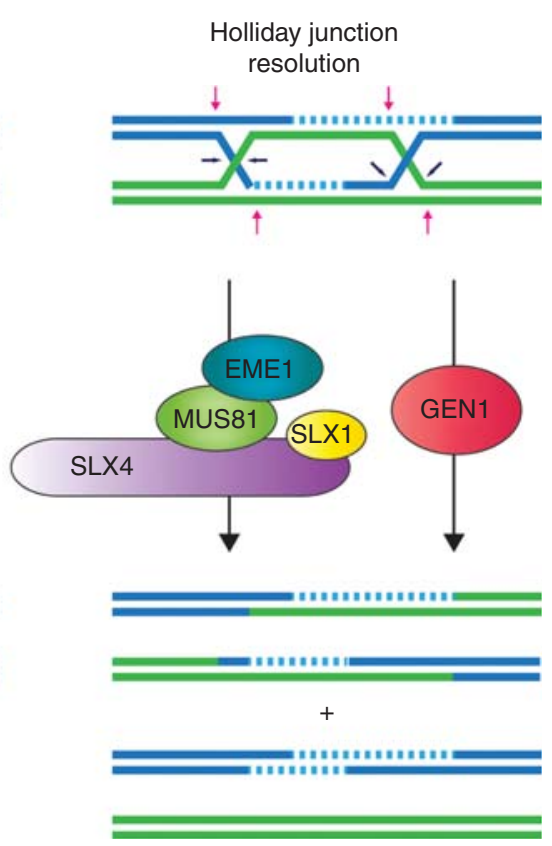

Crossover and noncrossover products Two distinct pathways and resolvases Essential in the absence of BLM

Figure 6. Holliday junction-processing enzymes in higher eukaryotes. In mitotic mammalian cells, the majority of junctions are removed by the BTR complex (BLM helicase, topoisomerase III $\alpha$, and RMI1/2; left panel). This dissolution reaction exclusively generates noncrossover products and is critical for the avoidance of loss of heterozygosity. However, mitotic cells also rely on structure-selective endonucleases to process HJs, particularly when the BTR pathway is compromised (e.g., in cells carrying BLM mutations). Three structure-selective endonucleases implicated in $\mathrm{HJ}$ processing comprise only two pathways of $\mathrm{HJ}$ resolution ( right panel). One pathway is mediated by the canonical HJ resolvase GEN1, whereas the second genetically distinct pathway involves the noncanonical resolvases SLX1-SLX4 and MUS81-EME1, which interact to form the SLX-MUS complex. 
rate-limiting incision and MUS81 introduces the second cut on the opposing strand of like polarity. Although this reaction is reminiscent of those catalyzed by canonical HJ resolvases, SLX-MUS promotes asymmetric cleavage of the junction and yields gapped and flapped DNA products that require further cellular processing (Fig. 6). Thus, SLX-MUS represents a noncanonical HJ resolvase that, intriguingly, appears to be specific for higher eukaryotes. Collectively, these studies provide novel insights into the nuclease activities of SLX-MUS and help to resolve the long-standing debate surrounding the $\mathrm{HJ}$ resolvase activity of MUS81EME1 (Haber and Heyer 2001).

\section{CONCLUDING REMARKS}

Homologous recombination is an essential process that conserves genetic information in somatic cells, while also promoting genetic diversity during meiosis. Central to the process of recombination is the formation and resolution of HJs, four-stranded DNA structures that physically connect two otherwise separated DNA helices. As such, HJs are potentially detrimental structures that need to be removed to allow for efficient DNA repair, accurate chromosome segregation, and maintenance of genome stability.

$\mathrm{HJ}$ resolvases are highly specialized endonucleases that exhibit a remarkable specificity for branched DNA substrates and, in particular, four-way DNA structures (i.e., HJs). Although junction-resolving enzymes were first identified in bacteriophages, the bacterial protein RuvC subsequently provided the paradigm for the identification of cellular $\mathrm{HJ}$ resolvases in higher organisms. However, this has been a slow and difficult task, partly because these enzymes lack primary sequence conservation. Nonetheless, junction-resolving enzymes have now been isolated from several different organisms, ranging from prokaryotes to mammals. The unifying property of these enzymes is their ability to convert the intact $\mathrm{HJ}$ into two disconnected DNA duplexes, in a metal-ion-dependent reaction called $\mathrm{HJ}$ resolution.

Intriguingly, $\mathrm{HJ}$ resolvases come in different flavors. Canonical HJ resolvases are defined as those proteins that exhibit biochemical similarity to RuvC. More specifically, these are homodimeric enzymes that use twin active sites to introduce two symmetrically opposed nicks across the helical junction. This reaction yields two nicked DNA duplexes that can be repaired by direct nick ligation. High-resolution structural data are available for several canonical $\mathrm{HJ}$ resolvases and have provided crucial insight into the mechanisms of $\mathrm{HJ}$ recognition and cleavage. However, eukaryotes also contain noncanonical $\mathrm{HJ}$ resolvases, which are heterodimeric enzymes that cleave HJs with asymmetric nicks. The products of these reactions are gapped and flapped DNA duplexes that cannot be directly ligated. Of particular interest are recent studies with the SLX-MUS enzyme, a noncanonical $\mathrm{HJ}$ resolvase in which the dual active sites for resolution are provided by two distinct structure-selective endonucleases. Our knowledge regarding the structure, biochemistry, and enzymology of such a eukaryotic noncanonical $\mathrm{HJ}$ resolvase remains limited, and this represents an important area of future research.

\section{ACKNOWLEDGMENTS}

We gratefully acknowledge our colleagues for their ideas, conversations, and advice, which have all contributed to the work discussed in this review. We also thank Miguel Gonzalez Blanco and Joao Matos for critical reading of the manuscript. Our apologies to those whose work we were unable to cite. Finally, thanks to Cancer Research UK, the Louis-Jeantet Foundation, the European Research Council, the Swiss Bridge Foundation, and the Breast Cancer Campaign for funding and support.

\section{REFERENCES}

* Reference is also in this collection

Abraham J, Lemmers B, Hande MP, Moynahan ME, Chahwan C, Ciccia A, Essers J, Hanada K, Chahwan R, Khaw AK, et al. 2003. Emel is involved in DNA damage processing and maintenance of genomic stability in mammalian cells. EMBO J 22: 6137-6147.

Agostinho A, Meier B, Sonneville R, Jagut M, Woglar A, Blow J, Jantsch V, Gartner A. 2013. Combinatorial regulation of meiotic Holliday junction resolution in 
C. elegans by HIM-6 (BLM) helicase, SLX-4, and the SLX-1, MUS-81 and XPF-1 nucleases. PLoS Genet 9: e1003591.

Andersen SL, Bergstralh DT, Kohl KP, LaRocque JR, Moore CB, Sekelsky J. 2009. Drosophila MUS312 and the vertebrate ortholog BTBD12 interact with DNA structure-specific endonucleases in DNA repair and recombination. Mol Cell 35: 128-135.

Andersen SL, Kuo HK, Savukoski D, Brodsky MH, Sekelsky J. 2011. Three structure-selective endonucleases are essential in the absence of BLM helicase in Drosophila. PLoS Genet 7: e1002315.

Ariyoshi M, Vassylyev DG, Iwasaki H, Nakamura H, Shinagawa H, Morikawa K. 1994. Atomic structure of the RuvC resolvase: A Holliday junction-specific endonuclease from E. coli. Cell 78: 1063-1072.

Ariyoshi M, Nishino T, Iwasaki H, Shinagawa H, Morikawa K. 2000. Crystal structure of the Holliday junction DNA in complex with a single RuvA tetramer. Proc Natl Acad Sci 97: 8257-8262.

Bastin-Shanower SA, Fricke WM, Mullen JR, Brill SJ. 2003. The mechanism of Mus81-Mms4 cleavage site selection distinguishes it from the homologous endonuclease Rad1-Rad10. Mol Cell Biol 23: 3487-3496.

Bennett RJ, West SC. 1995. Structural analysis of the RuvCHolliday junction complex reveals an unfolded junction. J Mol Biol 252: 213-226.

Bennett RJ, West SC. 1996. Resolution of Holliday junctions in genetic recombination: RuvC protein nicks DNA at the point of strand exchange. Proc Natl Acad Sci 93: 12217 12222.

Bennett RJ, Dunderdale HJ, West SC. 1993. Resolution of Holliday junctions by RuvC resolvase: Cleavage specificity and DNA distortion. Cell 74: 1021-1031.

Biertumpfel C, Yang W, Suck D. 2007. Crystal structure of T4 endonuclease VII resolving a Holliday junction. Nature 449: 616-620.

Birkenbihl RP, Kemper B. 1998. Endonuclease VII has two DNA binding sites each composed from one $\mathrm{N}$-terminus and one C-terminus provided by different subunits of the protein dimer. EMBO J 17: 4527-4534.

* Bizard AH, Hickson ID. 2014. The dissolution of double Holliday junctions. Cold Spring Harb Perspect Biol doi: 10.1101/cshperspect.a016477.

Blanco MG, Matos J, Rass U, Ip SCY, West SC. 2010. Functional overlap between the structure-specific nucleases Yen1 and Mus81-Mms4 for DNA damage repair in S. cerevisiae. DNA Repair 9: 394-402.

Boddy MN, Lopez-Girona A, Shanahan P, Interthal H, Heyer WD, Russell P. 2000. Damage tolerance protein Mus81 associates with the FHA1 domain of checkpoint kinase Cds1. Mol Cell Biol 20: 8758-8766.

Boddy MN, Gaillard PHL, McDonald WH, Shanahan P, Yates JR, Russell P. 2001. Mus81-Eme1 are essential components of a Holliday junction resolvase. Cell 107: 537548.

Bond CS, Kvaratskhelia M, Richard D, White MF, Hunter WN. 2001. Structure of Hjc, a Holliday junction resolvase, from Sulfolobus solfataricus. Proc Natl Acad Sci 98: 5509-5514.
Carlstrom G, Chazin WJ. 1996. Sequence dependence and direct measurement of crossover isomer distribution in model Holliday junctions using NMR spectroscopy. Biochemistry 35: 3534-3544.

Castor D, Nair N, Déclais AC, Lachaud C, Toth R, Macartney TJ, Lilley DMJ, Arthur JS, Rouse J. 2013. Cooperative control of Holliday junction resolution and DNA repair by the SLX1 and MUS81-EME1 nucleases. Mol Cell 52: 221-233.

Center M, Richardson C. 1970. An endonuclease induced after infection of Escherichia coli with bacteriophage T7. J Biol Chem 245: 6285-6292.

Ceschini S, Keeley A, McAlister MSB, Oram M, Phelan J, Pearl LH, Tsaneva IR, Barrett TE. 2001. Crystal structure of the fission yeast mitochondrial Holliday junction resolvase Ydc2. EMBO J 20: 6601-6611.

Chang JH, Kim JJ, Choi JM, Lee JH, Cho YJ. 2008. Crystal structure of the Mus81-Emel complex. Genes Dev 22: 1093-1106.

Chen JH, Churchill MAE, Tullius TD, Kallenbach NR, Seeman NC. 1988. Construction and analysis of monomobile DNA junctions. Biochemistry 27: 6032-6038.

Chen SM, Heffron F, Chazin WJ. 1993. Two-dimensional ${ }^{1} \mathrm{H}$ NMR studies of 32-base-pair synthetic immobile Holliday junctions: Complete assignments of the labile protons and identification of the base-pairing scheme. Biochemistry 32: 319-326.

Chen XB, Melchionna R, Denis CM, Gaillard PHL, Blasina A, Van de Weyer I, Boddy MN, Russell P, Vialard J, McGowan CH. 2001. Human MUS81-associated endonuclease cleaves Holliday junctions in vitro. Mol Cell 8: 11171127.

Chen L, Shi K, Yin Z, Aihara H. 2013. Structural asymmetry in the Thermus thermophilus RuvC dimer suggests a basis for sequential strand cleavages during Holliday junction resolution. Nucl Acids Res 41: 648-656.

Ciccia A, Constantinou A, West SC. 2003. Identification and characterization of the human Mus81/Eme1 endonuclease. J Biol Chem 278: 25172-25178.

Ciccia A, McDonald N, West SC. 2008. Structural and functional relationships of the XPF/MUS81 family of proteins. Annu Rev Biochem 77: 259-287.

Clegg RM, Murchie A, Zechel A, Carlberg C, Diekmann S, Lilley DMJ. 1992. Fluorescence resonance energy-transfer analysis of the structure of the four-way DNA junction. Biochemistry 31: 4846-4856.

Connolly B, West SC. 1990. Genetic recombination in Escherichia coli: Holliday junctions made by RecA protein are resolved by fractionated cell-free extracts. Proc Natl Acad Sci 87: 8476-8480.

Connolly B, Parsons CA, Benson FE, Dunderdale HJ, Sharples GJ, Lloyd RG, West SC. 1991. Resolution of Holliday junctions in vitro requires the Escherichia coli ruvC gene product. Proc Natl Acad Sci 88: 6063-6067.

Constantinou A, Davies AA, West SC. 2001. Branch migration and Holliday junction resolution catalyzed by activities from mammalian cells. Cell 104: 259-268.

Constantinou A, Chen X-B, McGowan CH, West SC. 2002. Holliday junction resolution in human cells: Two junction endonucleases with distinct substrate specificities. EMBO J 21: 5577-5585. 
H.D.M. Wyatt and S.C. West

Coulon S, Gaillard PHL, Chahwan C, McDonald WH, Yates JR, Russell P. 2004. Slx1-Slx4 are subunits of a structurespecific endonuclease that maintains ribosomal DNA in fission yeast. Mol Biol Cell 15: 71-80.

Coulon S, Noguchi E, Noguchi C, Du LL, Nakamura TM, Russell P. 2006. Rad22 $2^{\mathrm{Rad} 52}$-dependent repair of ribosomal DNA repeats cleaved by Slx1-Slx4 endonuclease. Mol Biol Cell 17: 2081-2090.

Crossan GP, van der Weyden L, Rosado IV, Langevin F, Gaillard PHL, McIntyre RE, Project SMG, Gallagher F, Kettunen MI, Lewis DY, et al. 2011. Disruption of mouse Slx4, a regulator of structure-specific nucleases, phenocopies Fanconi anemia. Nat Genet 43: 147-U199.

Daiyasu H, Komori K, Sakae S, Ishino Y, Toh H. 2000. Hjc resolvase is a distantly related member of the type II restriction endonuclease family. Nucl Acids Res 28: 4540-4543.

Davies AA, West SC. 1998. Formation of RuvABC-Holliday junction complexes in vitro. Curr Biol 8: 725-727.

Dayani Y, Simchen G, Lichten M. 2011. Meiotic recombination intermediates are resolved with minimal crossover formation during return-to-growth, an analog of the mitotic cell cycle. PLoS Genet 7: e1002083.

Déclais AC, Lilley DMJ. 2000. Extensive central disruption of a four-way junction on binding CCE1 resolving enzyme. J Mol Biol 296: 421-433.

Déclais AC, Lilley DMJ. 2008. New insight into the recognition of branched DNA structure by junction-resolving enzymes. Curr Opin Struct Biol 18: 86-95.

Déclais AC, Hadden J, Phillips SEV, Lilley DMJ. 2001. The active site of the junction-resolving enzyme T7 endonuclease I. J Mol Biol 307: 1145-1158.

Déclais AC, Fogg JM, Freeman ADJ, Coste F, Hadden JM, Phillips SEV, Lilley DMJ. 2003. The complex between a four-way DNA junction and T7 endonuclease I. EMBO J 22: $1398-1409$.

Déclais AC, Liu J, Freeman ADJ, Lilley DMJ. 2006. Structural recognition between a four-way DNA junction and a resolving enzyme. J Mol Biol 359: 1261-1276.

de los Santos T, Loidl J, Larkin B, Hollingsworth NM. 2001. A role for MMS4 in the processing of recombination intermediates during meiosis in Saccharomyces cerevisiae. Genetics 159: 1511-1525.

de Massy B, Weisberg RA, Studier FW. 1987. Gene 3 endonuclease of bacteriophage T7 resolves conformationally branched structures in double-stranded DNA. J Mol Biol 193: 359-376.

de Muyt A, Jessop L, Kolar E, Sourirajan A, Chen J, Dayani Y, Lichten M. 2012. BLM helicase ortholog Sgs1 is a central regulator of meiotic recombination intermediate metabolism. Mol Cell 46: 43-53.

Dendouga N, Gao H, Moechars D, Janicot M, Vialard J, McGowan CH. 2005. Disruption of murine Mus81 increases genomic instability and DNA damage sensitivity but does not promote tumorigenesis. Mol Cell Biol 25: 7569-7579.

Doe CL, Osman F, Dixon J, Whitby MC. 2000. The Holliday junction resolvase $S p C C E 1$ prevents mitochondrial DNA aggregation in Schizosaccharomyces pombe. Mol Gen Genet 263: 889-897.
Doe CL, Ahn JS, Dixon J, Whitby MC. 2002. Mus81-Eme1 and Rqh1 involvement in processing stalled and collapsed replication forks. J Biol Chem 277: 32753-32759.

Duckett DR, Murchie AIH, Diekmann S, Von Kitzing E, Kemper B, Lilley DMJ. 1988. The structure of the Holliday junction and its resolution. Cell 55: 79-89.

Duckett DR, Giraud-Panis MJE, Lilley DMJ. 1995. Binding of the junction-resolving enzyme T7 endonuclease I to DNA: Separation of binding and catalysis by mutation. $J$ Mol Biol 246: 95-107.

Dunderdale HJ, Benson FE, Parsons CA, Sharples GJ, Lloyd RG, West SC. 1991. Formation and resolution of recombination intermediates by E. coli RecA and RuvC proteins. Nature 354: 506-510.

Dunderdale HJ, Sharples GJ, Lloyd RG, West SC. 1994. Cloning, over-expression, purification and characterization of the Escherichia coli RuvC Holliday junction resolvase. J Biol Chem 269: 5187-5194.

Dunin-Horkawicz S, Feder M, Bujnicki JM. 2006. Phylogenomic analysis of the GIY-YIG nuclease superfamily. BMC Genomics 7: 98.

Eggleston AK, Mitchell AH, West SC. 1997. In vitro reconstitution of the late steps of genetic recombination in E. coli. Cell 89: 607-617.

Ehmsen KT, Heyer WD. 2008. Saccharomyces cerevisiae Mus81-Mms4 is a catalytic, DNA structure-selective endonuclease. Nucl Acids Res 36: 2182-2195.

Eichman BF, Vargason JM, Mooers BHM, Ho PS. 2000. The Holliday junction in an inverted repeat DNA sequence: Sequence effects on the structure of four-way junctions. Proc Natl Acad Sci 97: 3971-3976.

Elborough KM, West SC. 1990. Resolution of synthetic Holliday junctions in DNA by an endonuclease activity from calf thymus. EMBO J 9: 2931-2936.

Ezekiel UR, Zassenhaus HP. 1993. Localization of a cruciform cutting endonuclease to yeast mitochondria. Mol Gen Genet 240: 414-418.

Fabre F, Chan A, Heyer WD, Gangloff S. 2002. Alternate pathways involving Sgs1/Top3, Mus81/Mus81, and Srs2 prevent formation of toxic recombination intermediates from single-stranded gaps created by DNA replication. Proc Natl Acad Sci 99: 16887-16892.

Fekairi S, Scaglione S, Chahwan C, Taylor ER, Tissier A, Coulon S, Dong MQ, Ruse C, Yates JR, Russell P, et al. 2009. Human SLX4 is a Holliday junction resolvase subunit that binds multiple DNA repair/recombination endonucleases. Cell 138: 78-89.

Fogg JM, Lilley DMJ. 2000. Ensuring productive resolution by the junction-resolving enzyme RuvC: Large enhancement of the second-strand cleavage rate. Biochemistry 39: 16125-16134.

Fogg JM, Schofield MJ, White MF, Lilley DMJ. 1999. Sequence and functional-group specificity for cleavage of DNA junctions by RuvC of Escherichia coli. Biochemistry 38: $11349-11358$.

Fogg JM, Schofield MJ, Declais AC, Lilley DMJ. 2000. Yeast resolving enzyme CCE1 makes sequential cleavages in DNA junctions within the lifetime of the complex. Biochemistry 39: 4082-4089.

Fogg JM, Kvaratskhelia M, White MF, Lilley DMJ. 2001. Distortion of DNA junctions imposed by the binding 
of resolving enzymes: A fluorescence study. $J$ Mol Biol 313: $751-764$.

Frankel FR, Batcheler ML, Clark CK. 1971. The role of gene 49 in DNA replication and head morphogenesis in bacteriophage T4. J Mol Biol 62: 439-463.

Freeman AD, Declais AC, Lilley DM. 2013. The importance of the $\mathrm{N}$-terminus of $\mathrm{T} 7$ endonuclease $\mathrm{I}$ in the interaction with DNA junctions. J Mol Biol 425: 395-410.

Fricke WM, Brill SJ. 2003. Slx1-Slx4 is a second structurespecific endonuclease functionally redundant with Sgs 1Top3. Genes Dev 17: 1768-1778.

Fricke WM, Bastin-Shanower SA, Brill SJ. 2005. Substrate specificity of the Saccharomyces cerevisiae Mus81-Mms4 endonuclease. DNA Repair 4: 243-251.

Furukawa F, Kimura M, Ishibashi T, Mori Y, Hashimoto J, Sakaguchi K. 2003. OsSEND-1: A new RAD2 nuclease family member in higher plants. Plant Mol Biol 51: 5970 .

Gaillard P-HL, Noguchi E, Shanahan P, Russell P. 2003. The endogenous Mus81-Emel complex resolves Holliday junctions by a nick and couternick mechanism. Mol Cell 12: 747-759.

Gallo-Fernandez M, Sauger I, Ortiz-Bazan MA, Vazquez MV, Tercero JA. 2012. Cell cycle-dependent regulation of the nuclease activity of Mus81-Emel/Mms4. Nucl Acids Res 40: 8325-8335.

Gao M, Danielsen JR, Wei LZ, Zhou DP, Xu Q, Li MM, Wang ZQ, Tong WM, Yang YG. 2012. A novel role of human Holliday junction resolvase GEN1 in the maintenance of centrosome integrity. PLoS ONE 7: e49687.

Garner E, Kim Y, Lach FP, Kottemann MC, Smogorzewska A. 2013. Human GEN1 and the SLX4-associated nucleases MUS81 and SLX1 are essential for the resolution of replication-induced Holliday junctions. Cell Rep 5: $207-$ 215.

Giraud-Panis MJE, Lilley DMJ. 1996. T4 endonuclease VII: Importance of a histidine-aspartate cluster within the zinc-binding domain. J Biol Chem 271: 33148-33155.

Giraud-Panis MJE, Lilley DMJ. 1997. Near simultaneous DNA cleavage by the subunits of the junction-resolving enzyme T4 endonuclease VII. EMBO J 16: 2528-2534.

Golz S, Christoph A, Birkenkamp-Demtroder K, Kemper B. 1997. Identification of amino acids of endonuclease VII essential for binding and cleavage of cruciform DNA. Eur J Biochem 245: 573-580.

Gorecka KM, Komorowska W, Nowotny M. 2013. Crystal structure of RuvC resolvase in complex with Holliday junction substrate. Nucl Acids Res 41: 9945-9955.

Grainger RJ, Murchie AIH, Lilley DMJ. 1998. Exchange between stacking conformers in a four-way DNA junction. Biochemistry 37: 23-32.

Greger B, Kemper B. 1998. An apyrimidinic site kinks DNA and triggers incision by endonuclease VII of phage T4. Nucl Acids Res 26: 4432-4438.

Haber JE, Heyer WD. 2001. The fuss about Mus81. Cell 107: 551-554.

Hadden JM, Convery MA, Declais AC, Lilley DMJ, Phillips SEV. 2001. Crystal structure of the Holliday junction resolving enzyme T7 endonuclease I. Nat Struct Biol 8: $62-67$.
Hadden JM, Declais AC, Phillips SEV, Lilley DMJ. 2002. Metal ions bound at the active site of the junction-resolving enzyme T7 endonuclease I. EMBO J 21: 3505-3515.

Hadden JM, Declais AC, Carr SB, Lilley DMJ, Phillips SEV. 2007. The structural basis of Holliday junction resolution by T7 endonuclease. Nature 449: 621-624.

Hargreaves D, Rice DW, Sedelnikova SE, Artymiuk PJ, Lloyd RG, Rafferty JB. 1998. Crystal structure of E. coli RuvA with bound DNA Holliday junction at $6 \AA$ resolution. Nat Struct Biol 5: 441-446.

Ho CK, Mazón G, Lam AF, Symington LS. 2010. Mus81 and Yen1 promote reciprocal exchange during mitotic recombination to maintain genome integrity in budding yeast. Mol Cell 40: 988-1000.

Holliday R. 1964. A mechanism for gene conversion in fungi. Genet Res Camb 5: 282-304.

Holloway JK, Mohan S, Balmus G, Sun X, Modzelewski A, Borst PL, Freire R, Weiss RS, Cohen PE. 2011. Mammalian BTBD12 (SLX4) protects against genomic instability during mammalian spermatogenesis. PLoS Genet 7: e1002094.

Hyde H, Davies AA, Benson FE, West SC. 1994. Resolution of recombination intermediates by a mammalian endonuclease activity functionally analogous to Escherichia coli RuvC resolvase. J Biol Chem 269: 5202-5209.

Ichiyanagi K, Iwasaki H, Hishida T, Shinagawa H. 1998. Mutational analysis on structure-function relationship of a Holliday junction specific endonuclease RuvC. Genes Cells 3: 575-586.

Ingleston SM, Sharples GJ, Lloyd RG. 2000. The acidic pin of RuvA modulates Holliday junction binding and processing by the RuvABC resolvasome. EMBO J 19: 6266-6274.

Interthal H, Heyer WD. 2000. MUS81 encodes a novel helixhairpin-helix protein involved in the response to UV-and methylation-induced DNA damage in Saccharomyces cerevisiae. Mol Gen Genet 263: 812-827.

Ip SCY, Rass U, Blanco MG, Flynn HR, Skehel JM, West SC. 2008. Identification of Holliday junction resolvases from humans and yeast. Nature 456: 357-361.

Ishikawa G, Kanai Y, Takata K, Takeuchi R, Shimanouchi K, Ruike T, Furukawa T, Kimura S, Sakaguchi K. 2004. DmGEN, a novel RAD2 family endo-exonuclease from Drosophila melanogaster. Nucl Acids Res 32: 6251-6259.

Iwasaki H, Shiba T, Makino K, Nakata A, Shinagawa H. 1989. Overproduction, purification, and ATPase activity of the Escherichia coli RuvB protein involved in DNA repair. J Bacteriol 171: 5276-5280.

Iwasaki H, Takahagi M, Shiba T, Nakata A, Shinagawa H. 1991. Escherichia coli RuvC protein is an endonuclease that resolves the Holliday structure. EMBO J 10: 43814389.

Iwasaki H, Takahagi M, Nakata A, Shinagawa H. 1992. Escherichia coli RuvA and RuvB proteins specifically interact with Holliday junctions and promote branch migration. Genes Dev 6: 2214-2220.

Jensch F, Kemper B. 1986. Endonuclease VII resolves Y-junctions in branched DNA in vitro. EMBO J 5: 181-189.

Jessop L, Lichten M. 2008. Mus81/Mms4 endonuclease and Sgs 1 helicase collborate to ensure proper recombination intermediate metabolism during meiosis. Mol Cell 31: $313-323$. 
H.D.M. Wyatt and S.C. West

Johnson-Schlitz D, Engels WR. 2006. Template disruptions and failure of double Holliday junction dissolution during double-strand break repair in Drosophila BLM mutants. Proc Natl Acad Sci 103: 16840-16845.

Kaliraman V, Brill SJ. 2002. Role of SGS1 and SLX4 in maintaining rDNA structure in Saccharomyces cerevisiae. Curr Genet 41: 389-400.

Kaliraman V, Mullen JR, Fricke WM, Bastin-Shanower SA, Brill SJ. 2001. Functional overlap between Sgs1-Top3 and the Mms4-Mus81 endonuclease. Genes Dev 15: 27302740 .

Kemper B, Garabett M. 1981. Studies in T4-head maturation: 1. Purification and characterization of gene-49-controlled endonuclease. Eur J Biochem 115: 123-132.

Kemper B, Jensch F, Depka-Prondzynski M, Fritz HJ, Borgmeyer U, Mizuuchi K. 1984. Resolution of Holliday structures by endonuclease VII as observed in interactions with cruciform DNA. Cold Spring Harb Symp Quant Biol 49: 815-825.

Kim Y, Lach FP, Desetty R, Hanenberg H, Auerbach AD, Smogorzewska A. 2011. Mutations of the SLX4 gene in Fanconi anemia. Nat Genet 43: U142-U191.

Kleff S, Kemper B, Sternglanz R. 1992. Identification and characterization of yeast mutants and the gene for a cruciform cutting endonuclease. EMBO J 11: 699-704.

Komori K, Sakae S, Shinagawa H, Morikawa K, Ishino Y. 1999. A Holliday junction resolvase from Pyrococcus furiosus: Functional similarity to Escherichia coli RuvC provides evidence for conserved mechanism of homologous recombination in Bacteria, Eukarya, and Archaea. Proc Natl Acad Sci 96: 8873-8878.

Komori K, Sakae S, Daiyasu H, Toh H, Morikawa K, Shinagawa H, Ishino Y. 2000a. Mutational analysis of the $P y$ rococcus furiosus Holliday junction resolvase Hjc revealed functionally important residues for dimer formation, junction DNA binding, and cleavage activities. $J$ Biol Chem 275: 40385-40391.

Komori K, Sakae S, Fujikane R, Morikawa K, Shinagawa H, Ishino Y. 2000b. Biochemical characterization of the $\mathrm{Hjc}$ Holliday junction resolvase of Pyrococcus furiosus. Nucl Acids Res 28: 4544-4551.

Kvaratskhelia M, White MF. 2000a. An archaeal Holliday junction resolving enzyme from Sulfolobus solfataricus exhibits unique properties. J Mol Biol 295: 193-202.

Kvaratskhelia M, White MF. 2000b. Two Holliday junction resolving enzymes in Sulfolobus solfataricus. J Mol Biol 297: 923-932.

Kvaratskhelia M, Wardleworth BN, Norman DG, White MF. 2000. A conserved nuclease domain in the archaeal Holliday junction resolving enzyme Hjc. J Biol Chem 275: $25540-25546$.

* Lam I, Keeney S. 2014. Mechanism and regulation of meiotic recombination initiation. Cold Spring Harb Perspect Biol doi: 10.1101/cshperspect.a016634.

Lieber MR. 1997. The FEN-1 family of structure-specific nucleases in eukaryotic DNA replication, recombination and repair. BioEssays 19: 233-240.

Lilley DMJ. 2000. Structures of helical junctions in nucleic acids. Q Rev Biophys 33: 109-159.

Lilley DMJ, Kemper B. 1984. Cruciform-resolvase interactions in supercoiled DNA. Cell 36: 413-422.
Lockshon D, Zweifel SG, Freemancook LL, Lorimer HE, Brewer BJ, Fangman WL. 1995. A role for recombination junctions in the segregation of mitochondrial DNA in yeast. Cell 81: 947-955.

Lorenz A, West SC, Whitby MC. 2010. The human Holliday junction resolvase GEN1 rescues the meiotic phenotype of a Schizosaccharomyces pombe mus81 mutant. Nucl Acids Res 38: $1866-1873$.

Mankouri HW, Ashton TM, Hickson ID. 2011. Holliday junction-containing DNA structures persist in cells lacking Sgs1 or Top3 following exposure to DNA damage. Proc Natl Acad Sci 108: 4944-4949.

Manthei KA, Keck JL. 2014. The BLM dissolvasome in DNA replication and repair. Cell Mol Life Sci 70: 4067-4084.

Marrione PE, Cox MM. 1995. RuvB protein-mediated ATP hydrolysis: Functional asymmetry in the RuvB hexamer. Biochemistry 34: 9809-9818.

Matos J, Blanco MG, Maslen SL, Skehel JM, West SC. 2011. Regulatory control of the resolution of DNA recombination intermediates during meiosis and mitosis. Cell 147: $158-172$.

Matos J, Blanco MG, West SC. 2013. Cell cycle kinases coordinate the resolution of recombination intermediates with chromosome segregation. Cell Rep 4: 76-86.

McPherson JP, Lemmers B, Chahwan R, Pamidi A, Migon E, Maytysiak-Zablocki E, Moynahan ME, Essers J, Hanada K, Poonepalli A, et al. 2004. Involvement of mammalian Mus81 in genome integrity and tumor suppression. Science 304: $1822-1826$.

* Mehta A, Haber JE. 2014. Sources of DNA double-strand breaks and models for recombinational DNA repair. Cold Spring Harb Perspect Biol doi: 10.1101/cshperspect. a016428.

Middleton CL, Parker JL, Richard DJ, White MF, Bond CS. 2004. Substrate recognition and catalysis by the Holliday junction resolving enzyme Hje. Nucl Acids Res 32: $5442-$ 5451.

Miick SM, Fee RS, Millar DP, Chazin WJ. 1997. Crossover isomer bias is the primary sequence-dependent property of immobilized Holliday junctions. Proc Natl Acad Sci 94: 9080-9084.

Mitchell AH, West SC. 1994. Hexameric rings of Escherichia coli RuvB protein: Cooperative assembly, processivity, and ATPase activity. J Mol Biol 243: 208-215.

Mizuuchi K, Kemper B, Hays J, Weisberg RA. 1982. T4 endonuclease VII cleaves Holliday structures. Cell 29: $357-$ 365.

* Morrical SW. 2014. DNA pairing and annealing proceses in homologous recombination and homology-directed repair. Cold Spring Harb Perspect Biol doi: 10.1101/cshper spect.a016444.

Mueller JE, Kemper B, Cunningham RP, Kallenbach NR, Seeman NC. 1988. T4 endonuclease VII cleaves the crossover strands of Holliday junction analogs. Proc Natl Acad Sci 85: 9441-9445.

Mueller JE, Newton CJ, Jensch F, Kemper B, Cunningham RP, Kallenbach NR, Seeman NC. 1990. Resolution of Holliday junction analogs by T4 endonuclease VII can be directed by substrate structure. J Biol Chem 265: 13918-13924. 
Mullen JR, Kaliraman V, Ibrahim SS, Brill SJ. 2001. Requirement for three novel protein complexes in the absence of the Sgs1 DNA helicase in Saccharomyces cerevisiae. Genetics 157: 103-118.

Müller B, Jones C, West SC. 1990. T7 endonuclease I resolves Holliday junctions formed in vitro by RecA protein. $\mathrm{Nucl}$ Acids Res 18: 5633-5636.

Müller B, Tsaneva IR, West SC. 1993. Branch migration of Holliday junctions promoted by the Escherichia coli RuvA and RuvB proteins: II. Interaction of RuvB with DNA. J Biol Chem 268: 17185-17189.

Munoz IM, Hain K, Declais AC, Gardiner M, Toh GW, Sanchez-Pulido L, Heuckmann JM, Toth R, Macartney T, Eppink B, et al. 2009. Coordination of structure-specific nucleases by human SLX4/BTBD12 is required for DNA repair. Mol Cell 35: 116-127.

Murchie AIH, Lilley DMJ. 1993. T4 endonuclease VII cleaves DNA containing a cisplatin adduct. J Mol Biol 233: 77-85.

Murchie AIH, Clegg RM, von Kitzing E, Duckett DR, Diekmann S, Lilley DMJ. 1989. Fluorescence energy transfer shows that the four-way DNA junction is a right-handed cross of antiparallel molecules. Nature 341: 763-766.

Nishimoto H, Takayama M, Minagawa T. 1979. Purification and some properties of deoxyribonuclease whose synthesis is controlled by gene 49 of bacteriophage T4. Eur $J$ Biochem 100: 433-440.

Nishino T, Ariyoshi M, Iwasaki H, Shinagawa H, Morikawa K. 1998. Functional analyses of the domain structure in the Holliday junction binding protein RuvA. Structure 6: $11-21$.

Nishino T, Iwasaki H, Kataoka M, Ariyoshi M, Fujita T, Shinagawa H, Morikawa K. 2000. Modulation of RuvB function by the mobile domain III of the Holliday junction recognition protein RuvA. J Mol Biol 298: 407-416.

Nishino T, Komori K, Ishino Y, Morikawa K. 2001a. Dissection of the regional roles of the archaeal Holliday junction resolvase Hjc by structural and mutational analyses. J Biol Chem 276: 35735-35740.

Nishino T, Komori K, Tsuchiya D, Ishino Y, Morikawa K. 2001b. Crystal structure of the archaeal Holliday junction resolvase $\mathrm{Hjc}$ and implications for DNA recognition. Structure 9: 197-204.

Nishino T, Komori K, Ishino Y, Morikawa K. 2003. X-ray and biochemical anatomy of an archaeal XPF/Rad1/Mus81 family nuclease: Similarity between its endonuclease domain and restriction enzymes. Structure 11: 445-457.

Nowakowski J, Shim PJ, Prasad GS, Stout CD, Joyce GF. 1999. Crystal structure of an 82-nucleotide RNA-DNA complex formed by the 10-23 DNA enzyme. Nat Struct Biol 6: 151-156.

Nowotny M. 2009. Retroviral integrase superfamily: The structural perspective. EMBO Rep 10: 144-151.

Ögrünc M, Sancar A. 2003. Identification and characterization of human MUS81-MMS4 structure-specific endonuclease. J Biol Chem 278: 21715-21720.

Oh SD, Lao JP, Taylor AF, Smith GR, Hunter N. 2008. RecQ helicase, Sgs1, and XPF family endonuclease, Mus81$\mathrm{Mms} 4$, resolve aberrant joint molecules during meiotic recombination. Mol Cell 31: 324-336.
O’Neil NJ, Martin JS, Youds JL, Ward JD, Petalcorin MI, Rose AM, Boulton SJ. 2013. Joint molecule resolution requires the redundant activities of MUS-81 and XPF-1 during Caenorhabditis elegans meiosis. PLoS Genet 9: e1003582.

Oram M, Keeley A, Tsaneva I. 1998. Holliday junction resolvase in Schizosaccharomyces pombe has identical endonuclease activity to the Ccel homolog Ydc2. Nucl Acids Res 26: 594-601.

Ortiz-Lombardia M, Gonzalez A, Eritja R, Aymami J, Azorin F, Coll M. 1999. Crystal structure of a DNA Holliday junction. Nat Struct Biol 6: 913-917.

Overmars FJJ, Altona C. 1997. NMR study of the exchange rate between two stacked conformers of a model Holliday junction. J Mol Biol 273: 519-524.

Overmars FJ, Lanzotti V, Galeone A, Pepe A, Mayol L, Pikkemaat JA, Altona C. 1997. Design and NMR study of an immobile DNA four-way junction containing 38 nucleotides. Eur J Biochem 249: 576-583.

Parkinson MJ, Lilley DMJ. 1997. The junction resolving enzyme T7 endonuclease 1: Quaternary structure and interaction with DNA. J Mol Biol 270: 169-178.

Parkinson MJ, Pohler JRG, Lilley DMJ. 1999. Catalytic and binding mutants of the junction-resolving enzyme endonuclease I of bacteriophage T7: Role of acidic residues. Nucl Acids Res 27: 682-689.

Parsons CA, West SC. 1990. Specificity of binding to fourway junctions in DNA by bacteriophage T7 endonuclease I. Nucl Acids Res 18: 4377-4384.

Parsons CA, West SC. 1993. Formation of a RuvAB-Holliday junction complex in vitro. J Mol Biol 232: 397-405.

Parsons CA, Kemper B, West SC. 1990. Interaction of a fourway junction in DNA with T4 endonuclease VII. J Biol Chem 265: 9285-9289.

Parsons CA, Tsaneva I, Lloyd RG, West SC. 1992. Interaction of Escherichia coli RuvA and RuvB proteins with synthetic Holliday junctions. Proc Natl Acad Sci 89: 5452-5456.

Parsons CA, Stasiak A, Bennett RJ, West SC. 1995. Structure of a multisubunit complex that promotes DNA branch migration. Nature 374: 375-378.

Picksley SM, Parsons CA, Kemper B, West SC. 1990. Cleavage specificity of bacteriophage T4 endonuclease VII and bacteriophage T7 endonuclease I on synthetic branch migratable Holliday junctions. J Mol Biol 212: 723-735.

Pikkemaat JA, Van de Nelst H, Van Boom JH, Altona C. 1994. NMR studies and conformational analysis of a DNA four-way junction formed in a linear synthetic oligonucleotide. Biochemistry 33: 14896-14907.

Pöhler JRG, Giraud-Panis MJE, Lilley DMJ. 1996. T4 endonuclease VII selects and alters the structure of the fourway junction; binding of a resolution-defective mutant enzyme. J Mol Biol 260: 687-696.

Pottmeyer S, Kemper B. 1992. T4 endonuclease VII resolves cruciform DNA with nick and counter nick and its activity is directed by local nucleotide sequence. J Mol Biol 223: 607-615.

Raaijmakers H, Vix O, Toro I, Golz S, Kemper B, Suck D. 1999. X-ray structure of T4 endonuclease VII: A DNA junction resolvase with a novel fold and unusual domain-swapped dimer architecture. EMBO J 18: $1447-$ 1458 . 
H.D.M. Wyatt and S.C. West

Raaijmakers H, Toro I, Birkenbihl R, Kemper B, Suck D. 2001. Conformational flexibility in T4 endonuclease VII revealed by crystallography: Implications for substrate binding and cleavage. J Mol Biol 308: 311-323.

Rafferty JB, Sedelnikova SE, Hargreaves D, Artymiuk PJ, Baker PJ, Sharples GJ, Mahdi AA, Lloyd RG, Rice DW. 1996. Crystal structure of DNA recombination protein RuvA and a model for its binding to the Holliday junction. Science 274: 415-421.

Rass U, Compton SA, Matos J, Singleton MR, Ip SCY, Blanco MG, Griffith JD, West SC. 2010. Mechanism of Holliday junction resolution by the human GEN1 protein. Genes Dev 24: $1559-1569$.

Rodrigue A, Coulombe Y, Jacquet K, Gagne JP, Roques C, Gobeil S, Poirier G, Masson JY. 2013. The RAD51 paralogs ensure cellular protection against mitotic defects and aneuploidy. J Cell Sci 126: 348-359.

Roe SM, Barlow T, Brown T, Oram M, Keeley A, Tsaneva IR, Pearl LH. 1998. Crystal structure of an octameric RuvAHolliday junction complex. Mol Cell 2: 361-372.

Sadowski PD. 1971. Bacteriophage T7 endonuclease I: Properties of the enzyme purified from T7 phage infected Escherichia coli B. J Biol Chem 246: 209-216.

Saito A, Iwasaki H, Ariyoshi M, Morikawa K, Shinagawa H. 1995. Identification of four acidic amino acids that constitute the catalytic center of the RuvC Holliday junction resolvase. Proc Natl Acad Sci 92: 7470-7474.

Saito TT, Mohideen F, Meyer K, Harper JW, Colaiácovo MP. 2012. SLX-1 is required for maintaining genome integrity and promoting meiotic noncrossovers in the Caenorhabditis elegans germline. PLoS Genet 8: e1002888.

Saito TT, Lui DY, Kim H-M, Meyer K, Colaiácovo MP. 2013. Interplay between structure-specific endonucleases for crossover control during Caenorhabditis elegans meiosis. PLoS Genet 9: e1003586.

Sarbajna S, Davies D, West SC. 2014. Roles of SLX1-SLX4, MUS81-EME1, and GEN1 in avoiding genome instability and mitotic catastrophe. Genes Dev 28: 1124-1136.

Schofield MJ, Lilley DMJ, White MF. 1998. Dissection of the sequence specificity of the Holliday junction endonuclease Ccel. Biochemistry 37: 7733-7740.

Schwartz EK, Wright WD, Ehmsen KT, Evans JE, Stahlberg H, Heyer WD. 2012. Mus81-Mms4 functions as a single heterodimer to cleave nicked intermediates in recombinational DNA repair. Mol Cell Biol 32: 3065-3080.

Sha RJ, Iwasaki H, Liu FR, Shinagawa H, Seeman NC. 2000. Cleavage of symmetric immobile DNA junctions by Escherichia coli RuvC. Biochemistry 39: 11982-11988.

Shah R, Bennett RJ, West SC. 1994a. Activation of RuvC Holliday junction resolvase in vitro. $\mathrm{Nucl}$ Acids Res 22: 2490-2497.

Shah R, Bennett RJ, West SC. 1994b. Genetic recombination in E. coli: RuvC protein cleaves Holliday junctions at resolution hotspots in vitro. Cell 79: 853-864.

Shah R, Cosstick R, West SC. 1997. The RuvC dimer resolves Holliday junctions by a dual incision mechanism that involves base-specific contacts. EMBO J 16: 1464-1472.

Sharples GJ, Lloyd RG. 1991. Resolution of Holliday junctions in E. coli: Identification of the $r u v C$ gene product as a $19 \mathrm{kDa}$ protein. J Bacteriol 173: 7711-7715.
Sharples GJ, Benson FE, Illing GT, Lloyd RG. 1990. Molecular and functional analysis of the ruv region of Escherichia coli $\mathrm{K}-12$ reveals three genes involved in DNA repair and recombination. Mol Gen Genet 221: 219-226.

Shiba T, Iwasaki H, Nakata A, Shinagawa H. 1993. Escherichia coli RuvA and RuvB proteins involved in recombination repair: Physical properties and interactions with DNA. Mol Gen Genet 237: 395-399.

Shida T, Iwasaki H, Saito A, Kyogoku Y, Shinagawa H. 1996. Analysis of substrate specificity of the RuvC Holliday junction resolvase with synthetic Holliday junctions. $J$ Biol Chem 271: 26105-26109.

Sigal N, Alberts B. 1972. Genetic recombination: The nature of a crossed strand exchange between two homologous DNA molecules. J Mol Biol 71: 789-793.

Sigala B, Tsaneva IR. 2003. Functional dissection of the Schizosaccharomyces pombe Holliday junction resolvase Ydc2: In vivo role in mitochondrial DNA maintenance. Eur J Biochem 270: 2837-2847.

Smith GR, Boddy MN, Shanahan P, Russell P. 2003. Fission yeast Mus81-Emel Holliday junction resolvase is required for meiotic crossing over but not for gene conversion. Genetics 165: 2289-2293.

Stoepker C, Hain K, Schuster B, Hilhorst-Hofstee Y, Rooimans MA, Steltenpool J, Oostra AB, Eirich K, Korthof ET, Nieuwint AWM, et al. 2011. SLX4, a coordinator of structure-specific endonucleases, is mutated in a new Fanconi anemia subtype. Nat Genet 43: 138-141.

Svendsen JM, Smogorzewska A, Sowa ME, O'Connell BC, Gygi SP, Elledge SJ, Harper JW. 2009. Mammalian BTBD12/SLX4 assembles a Holliday junction resolvase and is required for DNA repair. Cell 138: 63-77.

* Symington LS. 2014. Processing of DNA braks: Mechanism and regulation. Cold Spring Harb Perspect Biol doi 10.1101/cshperspect.a016436.

Symington LS, Kolodner R. 1985. Partial purification of an enzyme from Saccharomyces cerevisiae that cleaves Holliday junctions. Proc Natl Acad Sci 82: 7247-7251.

Szakal B, Branzei D. 2013. Premature Cdk1/Cdc5/Mus81 pathway activation induces aberrant replication and deleterious crossover. EMBO J 32: 1155-1167.

Szostak JW, Orr-Weaver TL, Rothstein RJ, Stahl FW. 1983. The double-strand break repair model for recombination. Cell 33: 25-35.

Takahagi M, Iwasaki H, Nakata A, Shinagawa H. 1991. Molecular analysis of the Escherichia coli ruvC gene, which encodes a Holliday junction specific endonuclease. J Bacteriol 173: 5747-5753.

Takahagi M, Iwasaki H, Shinagawa H. 1994. Structural requirements of substrate DNA for binding to and cleavage by RuvC, a Holliday junction resolvase. J Biol Chem 269: 15132-15139.

Taylor ER, McGowan CH. 2008. Cleavage mechanism of human MUS81-EME1 acting on Holliday-junction structures. Proc Natl Acad Sci 105: 3757-3762.

Trowbridge K, McKim KS, Brill SJ, Sekelsky J. 2007. Synthetic lethality of Drosophila in the absence of the MUS81 endonuclease and the BLM helicase is associated with elevated apoptosis. Genetics 176: 1993-2001. 
Tsaneva IR, Illing GT, Lloyd RG, West SC. 1992a. Purification and properties of the RuvA and RuvB proteins of Escherichia coli. Mol Gen Genet 235: 1-10.

Tsaneva IR, Müller B, West SC. 1992b. ATP-dependent branch migration of Holliday junctions promoted by the RuvA and RuvB proteins of E. coli. Cell 69: 11711180.

Tsujimoto Y, Ogawa H. 1978. Intermediates in genetic recombination of bacteriophage T7 DNA. Biological activityand the roles of gene 3 and gene 5. J Mol Biol 125: 255-273.

van Gool AJ, Shah R, Mézard C, West SC. 1998. Functional interactions between the Holliday junction resolvase and the branch migration motor of Escherichia coli. EMBO J 17: $1838-1845$.

van Gool AJ, Hajibagheri NMA, Stasiak A, West SC. 1999. Assembly of the Escherichia coli RuvABC resolvasome directs the orientation of Holliday junction resolution. Genes Dev 13: 1861-1870.

Wechsler T, Newman S, West SC. 2011. Aberrant chromosome morphology in human cells defective for Holliday junction resolution. Nature 471: 642-646.

Wemmer DE, Wand AJ, Seeman NC, Kallenbach NR. 1985. NMR analysis of DNA junctions: Imino proton NMR studies of individual arms and intact junction. Biochemistry 24: 5745-5749.

West SC. 2009. The search for a human Holliday junction resolvase. Biochem Soc Trans 37: 519-526.

West SC, Korner A. 1985. Cleavage of cruciform DNA structures by an activity purified from Saccharomyces cerevisiae. Proc Natl Acad Sci 82: 6445-6449.

Whitby MC, Dixon J. 1997. A new Holliday junction resolving enzyme from Schizosaccharomyces pombe that is homologous to Ccel from Saccharomyces cerevisiae. J Mol Biol 272: 509-522.

White MF, Lilley DMJ. 1996. The structure-selectivity and sequence-preference of the junction-resolving enzyme CCE1 of Saccharomyces cerevisiae. J Mol Biol 257: 330-341.

White MF, Lilley DMJ. 1997a. Characterization of a Holliday junction-resolving enzyme from Schizosaccharomyces pombe. Mol Cell Biol 17: 6465-6471.
White MF, Lilley DMJ. 1997b. The resolving enzyme Ccel of yeast opens the structure of the four-way DNA junction. J Mol Biol 266: 122-134.

White MF, Lilley DMJ. 1998. Interaction of the resolving enzyme YDC2 with the four-way DNA junction. Nucl Acids Res 26: 5609-5616.

Wu F, Liu SY, Tao YM, Ou DP, Fang F, Yang LY. 2008. Decreased expression of methyl methansulfonate and ultraviolet-sensitive gene clone 81 (Mus81) is correlated with a poor prognosis in patients with hepatocellular carcinoma. Cancer 112: 2002-2010.

Wu F, Shirahata A, Sakuraba K, Kitamura Y, Goto T, Saito M, Ishibashi K, Kigawa G, Nemoto H, Sanada Y, et al. 2011. Downregulation of Mus81 as a novel prognostic biomarker for patients with colorectal carcinoma. Cancer Sci 102: 472-477.

Wyatt HDM, Sarbajna S, Matos J, West SC. 2013. Coordinated actions of SLX1-SLX4 and MUS81-EME1 for Holliday junction resolution in human cells. Mol Cell 52: 234-247.

Yang W, Lee JY, Nowotny M. 2006. Making and breaking nucleic acids: Two- $\mathrm{Mg}^{2+}$-ion catalysis and substrate specificity. Mol Cell 22: 5-13.

Yoshikawa M, Iwasaki H, Shinagawa H. 2001. Evidence that phenylalanine 69 in Escherichia coli RuvC resolvase forms a stacking interaction during binding and destabilization of a Holliday junction DNA substrate. J Biol Chem 276: 10432-10436.

Zakharyevich K, Tang SM, Ma YM, Hunter N. 2012. Delineation of joint molecule resolution pathways in meiosis identifies a crossover-specific resolvase. Cell 149: $334-$ 347.

Zhang C, Roberts TM, Yang J, Desai R, Brown GW. 2006. Suppression of genomic instability by SLX5 and SLX8 in Saccharomyces cerevisiae. DNA Repair 5: 336346.

* Zickler D, Kleckner N. 2014. Recombination, pairing, and synapsis of homologs during meiosis. Cold Spring Harb Perspect Biol doi: 10.1101/cshperspect. a016626. 


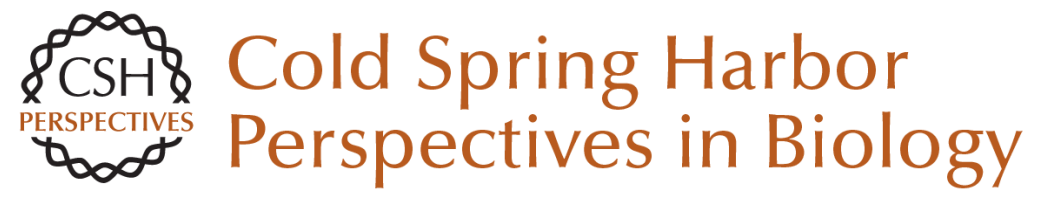

\section{Holliday Junction Resolvases}

Haley D.M. Wyatt and Stephen C. West

Cold Spring Harb Perspect Biol 2014; doi: 10.1101/cshperspect.a023192

Subject Collection DNA Recombination

Meiotic Recombination: The Essence of Heredity Neil Hunter

Regulation of Recombination and Genomic Maintenance Wolf-Dietrich Heyer

Initiation of Meiotic Homologous Recombination: Flexibility, Impact of Histone Modifications, and Chromatin Remodeling Lóránt Székvölgyi, Kunihiro Ohta and Alain Nicolas

Mechanism and Regulation of Meiotic

Recombination Initiation Isabel Lam and Scott Keeney

Homologous Recombination and Human Health: The Roles of BRCA1, BRCA2, and Associated Proteins

Rohit Prakash, Yu Zhang, Weiran Feng, et al.

\section{Cell Biology of Mitotic Recombination}

Michael Lisby and Rodney Rothstein

DNA-Pairing and Annealing Processes in

Homologous Recombination and

Homology-Directed Repair

Scott W. Morrical

Mediators of Homologous DNA Pairing

Alex Zelensky, Roland Kanaar and Claire Wyman
An Overview of the Molecular Mechanisms of

Recombinational DNA Repair

Stephen C. Kowalczykowski

Recombination, Pairing, and Synapsis of Homologs during Meiosis

Denise Zickler and Nancy Kleckner

DNA Strand Exchange and RecA Homologs in Meiosis

M. Scott Brown and Douglas K. Bishop

Meiosis and Maternal Aging: Insights from

Aneuploid Oocytes and Trisomy Births Mary Herbert, Dimitrios Kalleas, Daniel Cooney, et al.

Mismatch Repair during Homologous and Homeologous Recombination Maria Spies and Richard Fishel

Mechanisms of Gene Duplication and Amplification Andrew B. Reams and John R. Roth

The Role of Double-Strand Break Repair Pathways at Functional and Dysfunctional Telomeres Ylli Doksani and Titia de Lange

Regulation of DNA Pairing in Homologous Recombination James M. Daley, William A. Gaines, YoungHo Kwon, et al.

For additional articles in this collection, see http://cshperspectives.cshlp.org/cgi/collection/

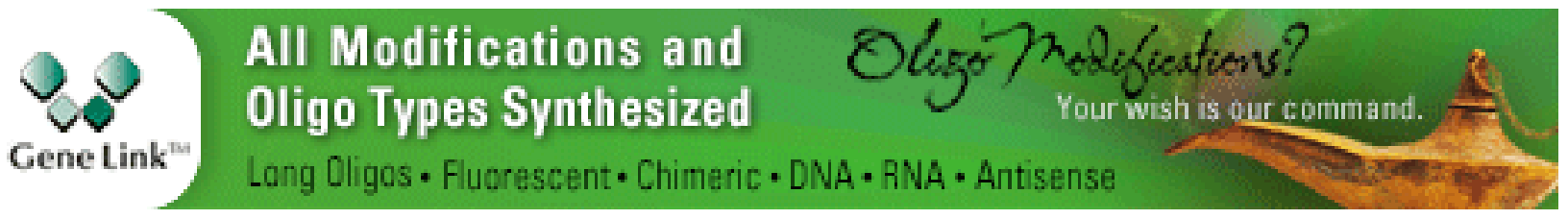


For additional articles in this collection, see http://cshperspectives.cshlp.org/cgi/collection/

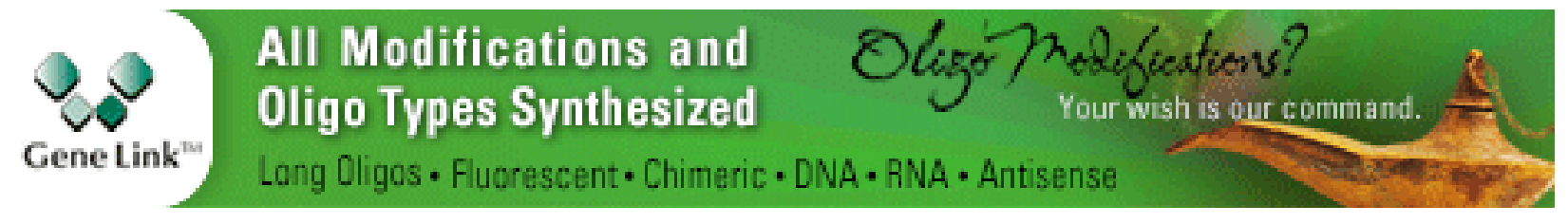

Copyright @ 2014 Cold Spring Harbor Laboratory Press; all rights reserved 